\title{
ON THE GLOBAL STRENGTH ANALYSIS OF PRELIMINARY DESIGN FOR SEVERAL FLOATING DOCK TYPES
}

\author{
Article DOI: https://doi.org/10.35219/mtd.2019.1.01 \\ Elisabeta BURLACU, Leonard DOMNISORU \\ "Dunărea de Jos" University of Galati, Romania \\ email: leonard.domnisoru@ugal.ro
}

\begin{abstract}
The design concepts of floating docks used for shipyards launching activities have to be assessed from the preliminary design phases by global strength criteria. At initial design, few technical data of floating docks are available, so that the global strength analysis can be developed only on simplified numerical models by the hull equivalent beam method. As loading conditions several docking cases and equivalent design waves, head and oblique conditions, are considered, according to the floating dock design rules. The computations are done with own codes, involving non-linear iterative procedures, which require as input data the $3 D$-CAD floating docks offset lines and on-board mass distributions. For studying floating dock models, the authors have considered three constructive versions: small dock with continuous and non-continuous side ballast tanks, large dock resulting from a conversion of an off-shore barge. The global strength criteria are the admissible sectional efforts by design rules of statistical values and the ultimate strength vertical bending moment by Smith method. The free board operation criterion is also considered for safety evaluation. The preliminary operation limits of the analyzed floating docks are obtained, in terms of equivalent design wave height, pointing out the main differences between the three constructive versions included in this study.
\end{abstract}

Keywords: preliminary design, floating docks, global strength, design wave, equivalent beam model

\section{INTRODUCTION}

At preliminary design phase of a floating dock structure [9], [10], one of the initial evaluations is the global strength by simplified hull equivalent beam method [8], under several operation cases, according to the docks' design rules [4], [5].

This study includes comparative analyses between three floating docks constructive versions, having either different ballast tanks layout on the main deck or lifting capacity [2], [3]. The numerical analyses are developed with own and rules codes (Fig. 1), suitable for the initial design phase [3], [5], [6]. For the floating docks, several operation scenarios are considered, light case, transit state docking operation, maximum lifting capacity loading, according to the design rules [4], [5] and shipyards' docking processes [2].

For each floating dock version, besides the still water condition, also equivalent design wave's loads [8], [9], head or oblique, are considered. Although the equivalent beam model uses a reduced set of input data, typical for preliminary design, the strength of the floating docks can be assessed by design global bending and torsion moments, shear forces and ultimate vertical bending moment criteria [4], [5], [8], [9]. Before any strength assessment, the free-board criterion is also assessed [1].

This study delivers as results the preliminary evaluation of the floating docks operational capabilities in terms of design wave's height limits and makes possible to compare three floating docks constructive versions, which are used in the shipyards docking operations [7].

\section{FUNDAMENTS ON GLOBAL STRENGHT ANALYSIS BY EQUIVALENT BEAM MODELS}

For the global strength analysis by equivalent beam models [8] under equivalent design waves EDW [9], the authors have developed two codes: D_ACAVD [3] in the case of head and follow design waves, P_QSWD [6] in the case of oblique design waves, with the linked logical flowchart presented in Fig. 1. 


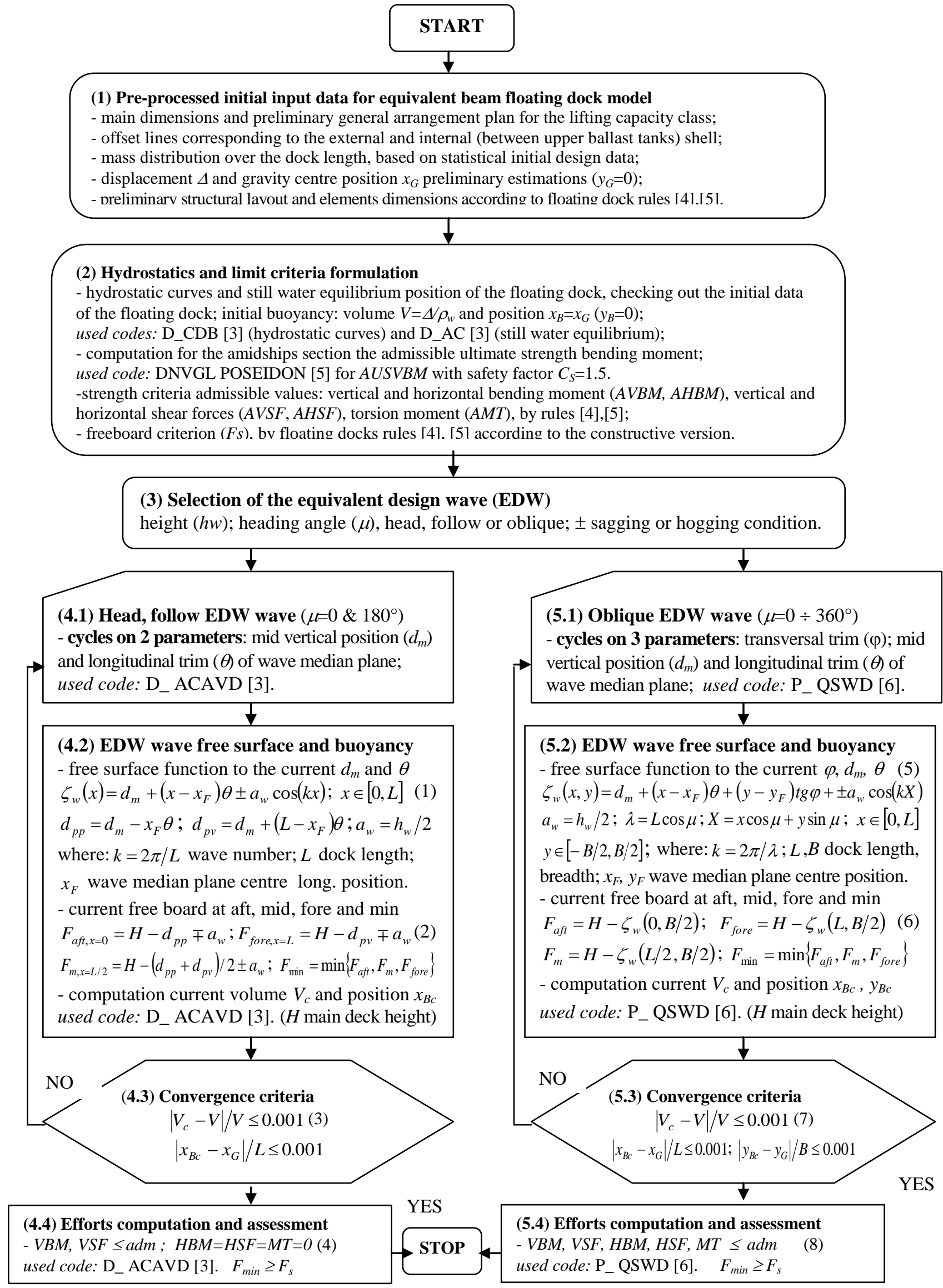

Fig. 1. Logical flowchart of global strength assessment by equivalent beam floating dock models 
Module 1 includes the floating docks initial data pre-processing and the equivalent beam model development.

Module 2 involves own codes D_CDB, D_AC [3] for floating docks hydrostatics computation and equilibrium in still water condition $\left(h_{w}=0\right)$. The ultimate strength vertical bending moment USVBM (global buckling) is computed by DNVGL POSEIDON [5] code, for the preliminary structural scantlings of the floating docks. Also in this module the admissible values for sectional efforts and freeboard criterion are selected by floating docks design rules [4], [5].

Module 3 ma kes the selection of the equivalent design wave EDW parameters, switching for one of the numerical codes.

Modules 4.1-4, equations (1)-(4), present in brief the main steps for the two parameters non-linear iterative process for balance between the floating dock hull and head (follow) equivalent design wave by D_ACAVD [3] code.

Modules 5.1-4, equations (5)-(8), present in brief the main steps for the three parameters non-linear iterative process for balance between the floating dock hull and oblique equivalent design wave by P_QSWD [6].

More details for the theoretical bases of algorithms from Fig.1 are presented in the selected references [3], [6].

\section{THE FLOATING DOCKS STUDY CASES CHARACTERISTICS}

As study cases we have considered three floating docks constructive cases, in order to analyze the main differences that occur from the point of strength criteria at initial design phase.

The first floating dock NWT-Dock60 is small size, length of $60 \mathrm{~m}$, maximum lifting capacity $828 \mathrm{t}$, with non-continuous side water ballast tanks [3]. Table 1 presents the main data of the NWT-Dock60 equivalent beam dock model. Figures $2 \mathrm{a}$ and $\mathrm{b}$ present the NWT-Dock60 model under equivalent design waves with $h_{w}=1.278 \mathrm{~m}$, sagging and hogging, quarter-sea $\mu=45^{\circ}$ heading angle. In order to increase the global ultimate strength (US) capacity, very limited for the initial structure [3], we have reconsidered the frames distance $a_{F r}=a_{0}=600 \mathrm{~mm}$ instead of $a_{F r}=2 a_{0}=1200 \mathrm{~mm}$. Figure $3 \mathrm{~b}$ presents the ultimate strength vertical bending moment USVBM by Smith method [5] for NWT-Dock60 with amidships initial structure from Fig. 3a. At aft and fore peak, where the side ballast tanks are placed above the main deck, the transversal structure becomes similar to the second floating dock (Fig. 5a). For NWT-Dock60 are considered the following loading cases: light without docking mass, ship 1 with uniform docking mass, ship 2 with sagging docking mass and ship 3 with hogging mass distribution, according to dock design rules [5]. See mass distributions in Figures $6 a$ and $b$.

The second floating dock CWT-Dock60 is small size, length of $60 \mathrm{~m}$, maximum lifting capacity $828 \mathrm{t}$, with continuous side water ballast tanks caisson type [3], [5]. The main data of the CWT-Dock60 equivalent beam dock model are in Table 1. Figs.4.a.b presents the CWT-Dock60 model under equivalent design wave with $h_{w}=1.930 \mathrm{~m}$, sagging and hogging, quartersea $\mu=45^{\circ}$ heading angle. Similar to the first dock NWT-Dock60, so that we have a same structural reference, the frames distance is reconsidered at $a_{F r}=a_{0}=600 \mathrm{~mm}$ instead of $a_{F r}=2 a_{0}=1200 \mathrm{~mm} \mathrm{[3].}$ Fig.5.b presents the ultimate strength vertical bending moment USVBM by Smith method [5] for CWTDock60 with amidships initial structure from Fig.5.a. For CWT-Dock60 are considered same four mass distribution cases, with diagrams not included in this paper.

The third floating dock DOCKV is large size, length of $209.2 \mathrm{~m}$, maximum lifting capacity $27000 \mathrm{t}$, with non-continuous side water ballast tanks [2]. A selection of the main data of the DOCKV equivalent beam dock model is presented in Table 1. Figs. 7.a.b presents the DOCKV model under equivalent design wave with $h_{w}=4.492 \mathrm{~m}$, sagging and hogging, head wave $\mu=0$ condition. The frames distance is $a_{F r}=4 a_{0}=3000 \mathrm{~mm}$ [2]. Figure $8 \mathrm{~b}$ presents the ultimate strength vertical bending moment USVBM by Smith method [5] for DOCKV with amidships initial structure from Fig. 8a. The following DOCKV loading cases are considered: light case without docking mass and with ballast, D19747 $\mathrm{t}$ with shipyard docking test mass [2], D27000 t (unif.) with uniform docking mass, D27000 t (hogg.) with hogging mass distribution and D27000 t (sagg.) with sagging docking mass, according to dock design rules [4], [5]. See mass distributions in Figures 9a, b, c. In order to have the quay transfer required main deck level, the on-board ballast system ensures a draught constant level of $T_{m}=6.2 \mathrm{~m}$ for all docking cases.

Table 2 presents the global strength admissible values, based on dock design rules [4], [5], for VBM $[\mathrm{kNm}]$ vertical bending moment, VSF $[\mathrm{kN}]$ vertical shear force, $H B M[\mathrm{kNm}]$ horizontal bending moment, $H S F[\mathrm{kN}]$ horizontal shear force, $M T[\mathrm{kNm}]$ torsion moment, including USVBM $[\mathrm{kNm}]$ ultimate strength vertical moment and freeboard $F_{s}$ [m] criteria.

The floating docks are analyzed up to the maximum EDW equivalent design wave height [5], corresponding to the maximum river class $\operatorname{IN}(2.0)$ and costal class $\mathrm{RE}(50 \%)$ for the floating docks operation water areas conditions. 
Table 1. The floating docks study cases main characteristics [2], [3]

\begin{tabular}{|c|c|c|c|c|}
\hline \multirow{2}{*}{\multicolumn{2}{|c|}{\begin{tabular}{|l} 
Floating dock version \\
Side water ballast tanks type
\end{tabular}}} & NWT-Dock60 (Figs.2.a,b) & CWT-Dock60 (Figs.4.a,b) & DOCKV (Figs.7.a,b) \\
\hline & & Non-continuous & Continuous & Non-continuous \\
\hline$M_{\text {lift }}[t]$ & maximum lifting mass & 828 & 828 & 27000 \\
\hline$L[\mathrm{~m}]$ & length & 60 & 60 & 209.2 \\
\hline$B[\mathrm{~m}]$ & breadth & 20 & 20 & 55.13 \\
\hline$B_{\max }[\mathrm{m}]$ & maximum breadth & 20 & 20 & 61.09 \\
\hline$H[\mathrm{~m}]$ & pontoon deck height & 2 & 2 & 10.10 \\
\hline$a_{0}[\mathrm{~mm}]$ & regular distance & 600 & 600 & 750 \\
\hline$a_{F r}[\mathrm{~mm}]$ & frames distance & 600 & 600 & 3000 \\
\hline$N S$ & number of stations beam model & 300 & 300 & 280 \\
\hline$z_{N}[\mathrm{~m}]$ & neutral axis vertical position & 1.000 & 2.728 & 5.139 \\
\hline$z_{R}[\mathrm{~m}]$ & torsion centre vertical position & 1.000 & -1.168 & 5.139 \\
\hline \multicolumn{2}{|c|}{ Material type } & steel grade $\mathrm{A}$ & steel grade $\mathrm{A}$ & steel grade $\mathrm{AH} 36$ \\
\hline$h_{w \max }[\mathrm{m}]$ & max. wave height RE(50\%) & 2.568 & 2.568 & 4.492 \\
\hline$\mu\left[{ }^{\circ}\right]$ & EDW heading angle & $0 \div 360$ oblique & $0 \div 360$ oblique & 0 head (180 follow) \\
\hline \multirow{2}{*}{$\Delta[\mathrm{t}]$} & \multirow{2}{*}{ displacement } & 960 & 1152 & 66324 \\
\hline & & 1788 & 1980 & 66324 \\
\hline$x_{G}[\mathrm{~m}]$ & gravity centre long. position & 30 & 30 & 100.148 \\
\hline$y_{G}[\mathrm{~m}]$ & gravity centre vert. position & 0 & 0 & 0 \\
\hline \multirow{2}{*}{$T_{m}[\mathrm{~m}]$} & \multirow{2}{*}{$\begin{array}{l}\text { medium draught in } \\
\text { still water }\end{array}$} & 0.800 & 0.960 & 6.200 \\
\hline & & 1.490 & 1.650 & 6.200 \\
\hline$\theta$ [rad] & longitudinal trim in still water & 0 & 0 & 0 \\
\hline$\varphi[\mathrm{rad}]$ & transversal trim in still water & 0 & 0 & 0 \\
\hline
\end{tabular}

Table 2. The admissible values for global strength equivalent beam model formulation for floating docks versions [4],[5]

\begin{tabular}{|c|c|c|c|c|c|c|c|c|c|}
\hline $\begin{array}{c}\text { Floating } \\
\text { dock } \\
\text { version }\end{array}$ & $\begin{array}{c}\text { USVBM } \\
{[\mathrm{kNm}]} \\
\text { ultimate }\end{array}$ & $\begin{array}{c}\text { AUSVBM } \\
{[\mathrm{kNm}]} \\
(c s=1.5)\end{array}$ & $\begin{array}{c}\text { VBM-adm } \\
{[\mathrm{kNm}]} \\
\text { rules }\end{array}$ & $\begin{array}{c}\text { AVBM } \\
{[\mathrm{kNm}]} \\
\text { combined }\end{array}$ & $\begin{array}{c}\text { AVSF } \\
{[\mathrm{kN}]} \\
\text { rules }\end{array}$ & $\begin{array}{c}\text { AHBM } \\
{[\mathrm{kNm}]} \\
\text { rules }\end{array}$ & $\begin{array}{c}\text { AHSF } \\
{[\mathrm{kN}]} \\
\text { rules }\end{array}$ & $\begin{array}{c}\text { AMT } \\
{[\mathrm{kNm}]} \\
\text { rules }\end{array}$ & $\begin{array}{c}\text { Fs } \\
{[\mathrm{m}]} \\
\text { rules }\end{array}$ \\
\hline NWT-Dock60 & $3.41 \mathrm{E}+04$ & $2.27 \mathrm{E}+04$ & $5.56 \mathrm{E}+04$ & $2.27 \mathrm{E}+04$ & $3.14 \mathrm{E}+03$ & $4.26 \mathrm{E}+03$ & $2.11 \mathrm{E}+02$ & $2.44 \mathrm{E}+04$ & 0.300 \\
\hline CWT-Dock60 & $9.48 \mathrm{E}+04$ & $6.32 \mathrm{E}+04$ & $5.56 \mathrm{E}+04$ & $5.56 \mathrm{E}+04$ & $3.14 \mathrm{E}+03$ & $5.11 \mathrm{E}+03$ & $2.54 \mathrm{E}+02$ & $2.44 \mathrm{E}+04$ & 0.075 \\
\hline DOCKV & $7.97 \mathrm{E}+06$ & $5.32 \mathrm{E}+06$ & $3.44 \mathrm{E}+06$ & $3.44 \mathrm{E}+06$ & $5.70 \mathrm{E}+04$ & $8.62 \mathrm{E}+05$ & $1.23 \mathrm{E}+04$ & $1.08 \mathrm{E}+06$ & 0.300 \\
\hline
\end{tabular}

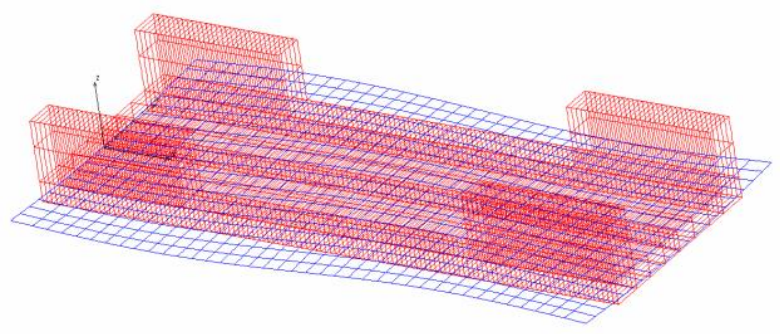

Fig. 2a. NWT-Dock60, light case, EDW wave hogging, Fig. 2b. NWT-Dock60, light case, EDW wave sagging, $h_{w}=1.278 \mathrm{~m}$, quarter-sea $\mu=45^{\circ}$, and offset lines [3]

CL

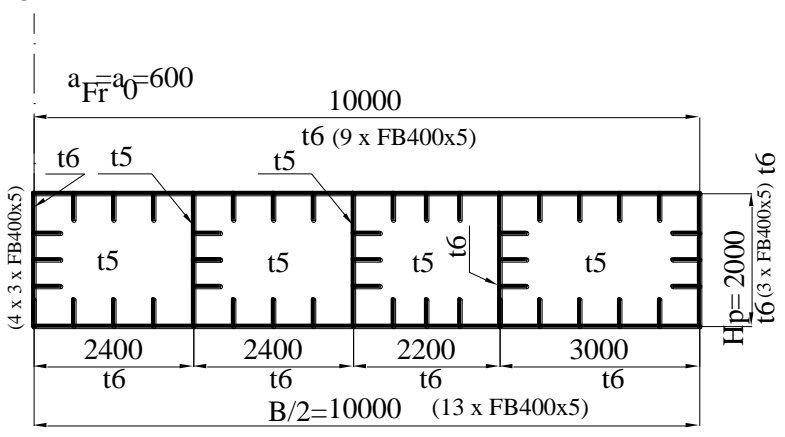

Fig. 3a. NWT-Dock60 amidships transversal floating dock preliminary structure [3]

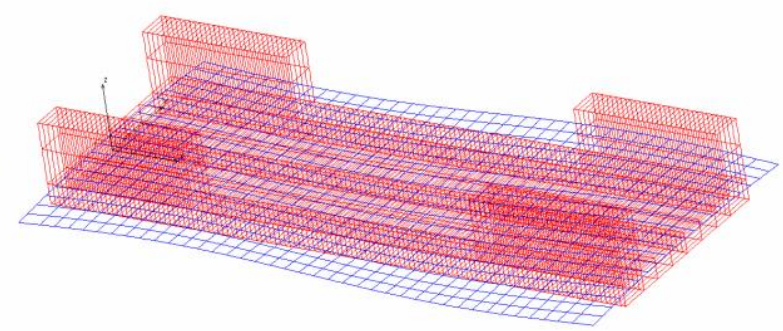

$h_{w}=1.278 \mathrm{~m}$, quarter-sea $\mu=45^{\circ}$, and offset lines [3]

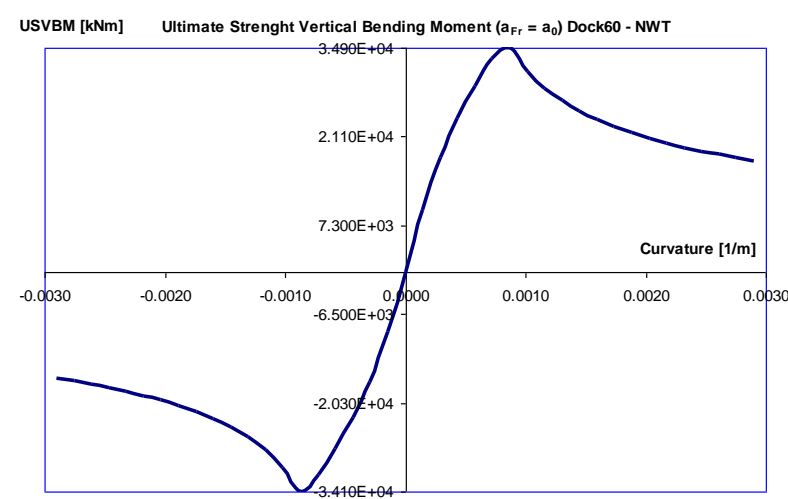

Fig. 3b. NWT-Dock60 ultimate strength vertical bending moment USVBM $[\mathrm{kNm}], a_{F r}=a_{0}=600 \mathrm{~mm}$ 

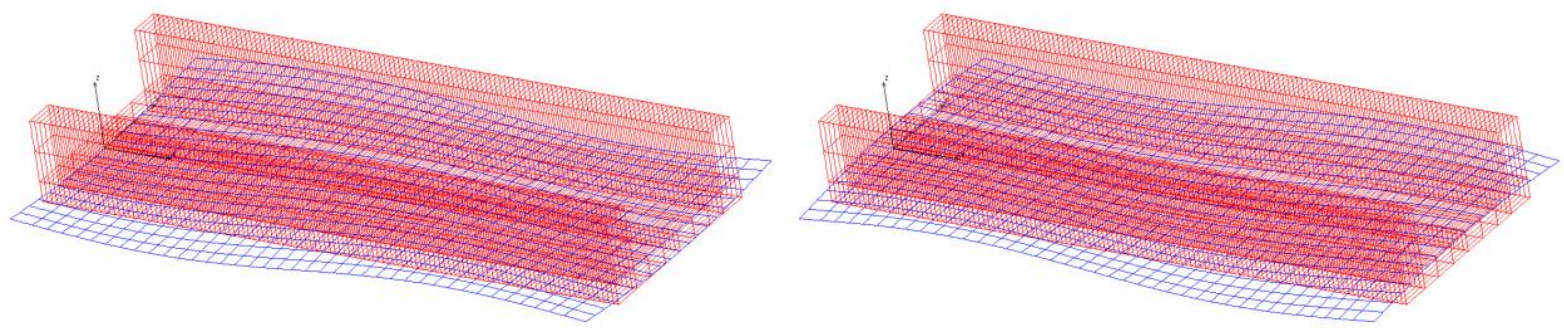

Fig. 4a. CWT-Dock60, light case, EDW wave hogging, Fig. 4b. CWT-Dock60, light case, EDW wave sagging, $h_{w}=1.930 \mathrm{~m}$, quarter-sea $\mu=45^{\circ}$, and offset lines [3] $\quad h_{w}=1.930 \mathrm{~m}$, quarter-sea $\mu=45^{\circ}$, and offset lines [3]

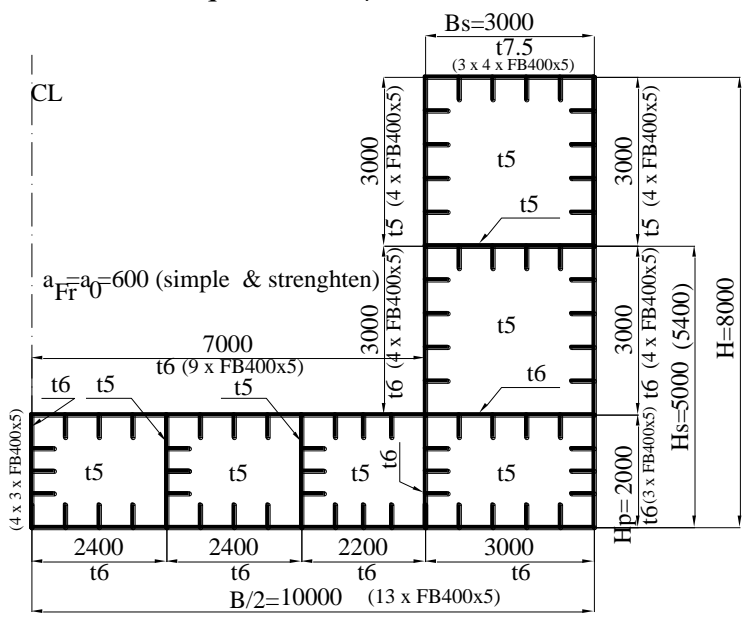

Fig. 5a CWT-Dock60 amidships transversal floating dock preliminary structure [3]

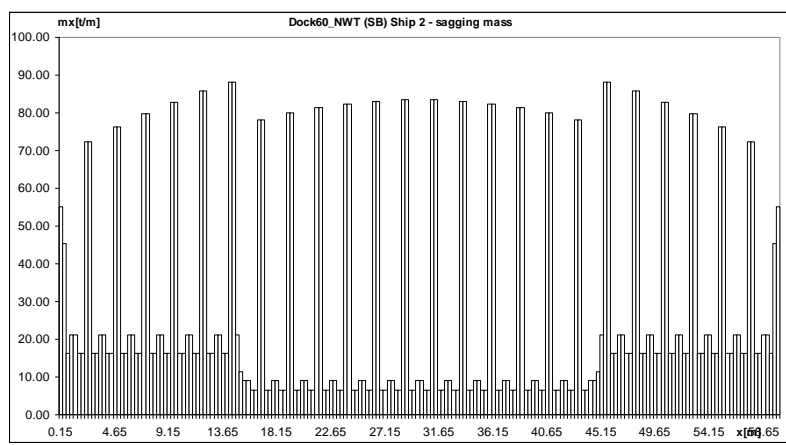

Fig. 6a. NWT-Dock60, ship 2 case, with sagging docking mass distribution [3]

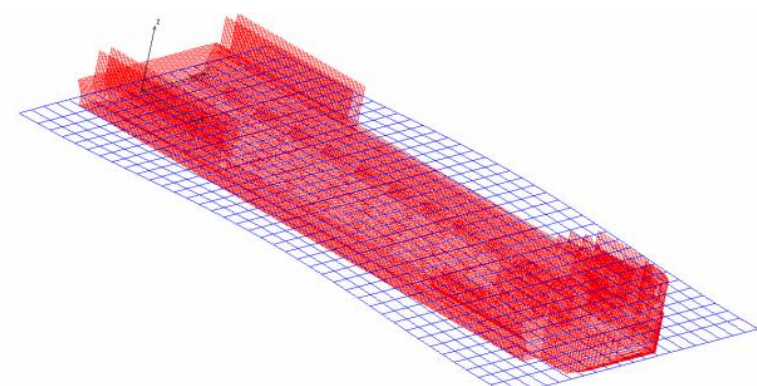

Fig.7a. DOCKV, light case, EDW wave hogging, $h_{w}=4.492 \mathrm{~m}$, head $\mu=0$ condition, and offset lines [2]

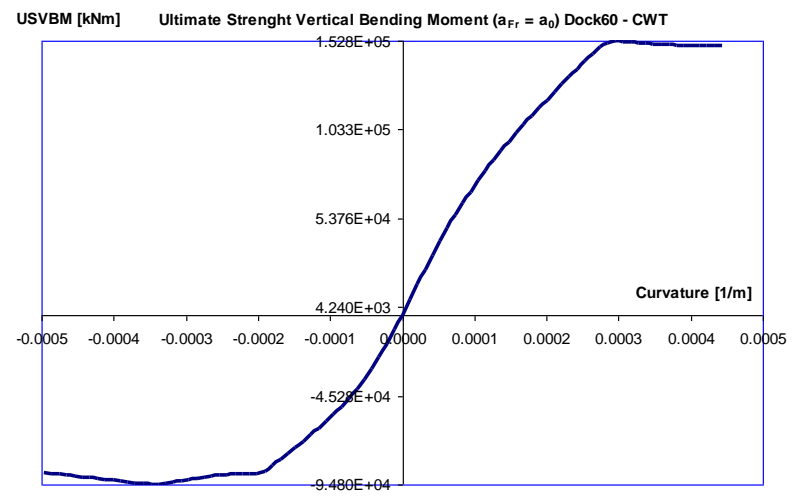

Fig. 5b CWT-Dock60 ultimate strength vertical bending moment USVBM $[\mathrm{kNm}], a_{F r}=a_{0}=600 \mathrm{~mm}$

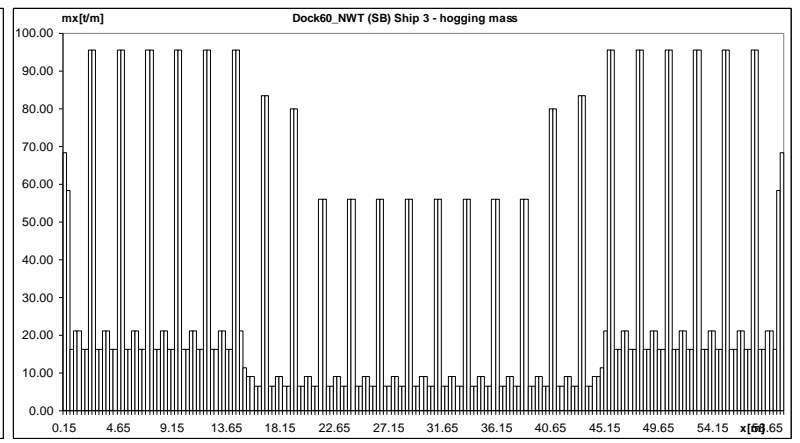

Fig. 6b. NWT-Dock60, ship 3 case, with hogging docking mass distribution [3]

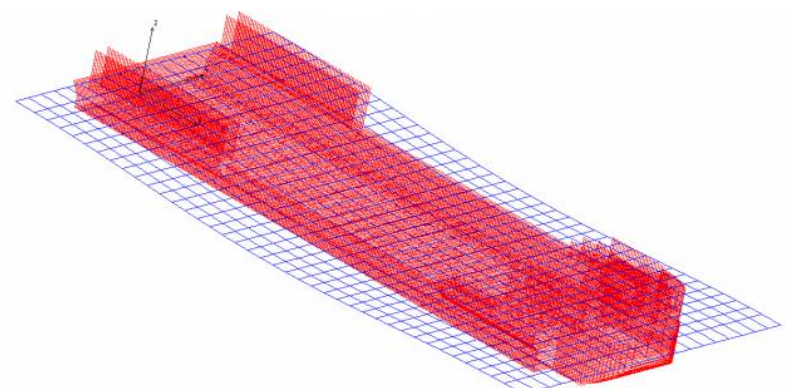

Fig. 7b. DOCKV, light case, EDW wave sagging, $h_{w}=4.492 \mathrm{~m}$, head $\mu=0$ condition, and offset lines [2] 


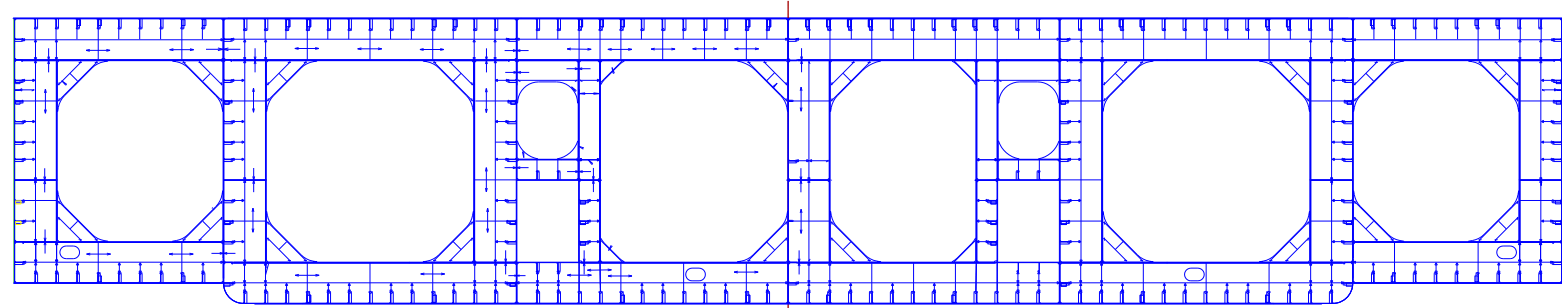

Fig. 8a DOCKV amidships transversal floating dock structure [2]

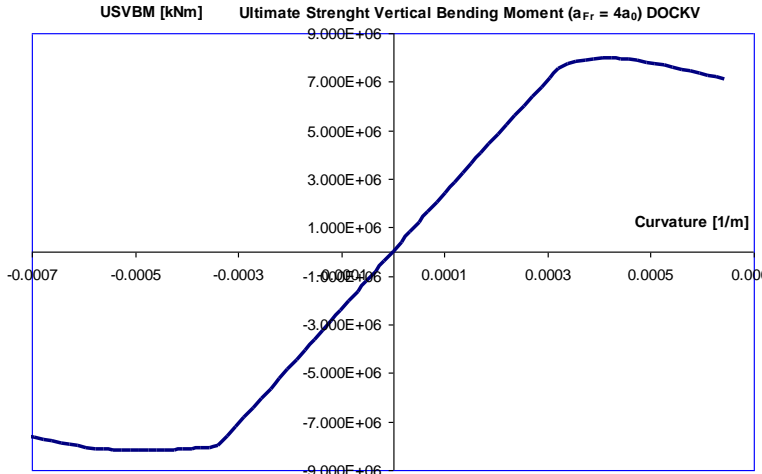

Fig. 8b. DOCKV ultimate strength vertical bending moment USVBM $[\mathrm{kNm}], a_{F r}=4 a_{0}=3000 \mathrm{~mm}$

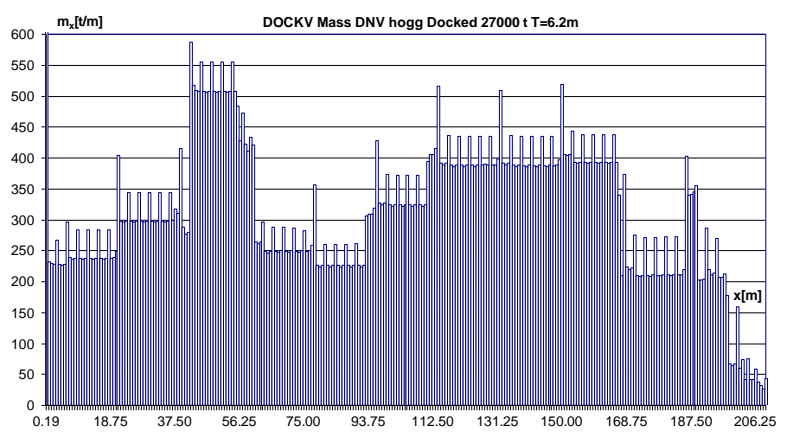

Fig. 9b. DOCKV D27000t (\&ball) hogg-mass, with hogging docking mass distribution, $T_{m}=6.2 \mathrm{~m}$ [2]

\section{FLOATING DOCKS NWT-DOCK60 AND CWT-DOCK60 GLOBAL STRENGHT ANALYSIS IN OBLIQUE WAVES, BY NUMERICAL EQUIVALENT DOCK BEAM MODELS}

In the cases of oblique equivalent design waves, taking into account that the NWT-Dock60 and CWTDock60 floating docks have double symmetry planes (Figs. 2a, b and Figs. 4a, b), the heading angle can be

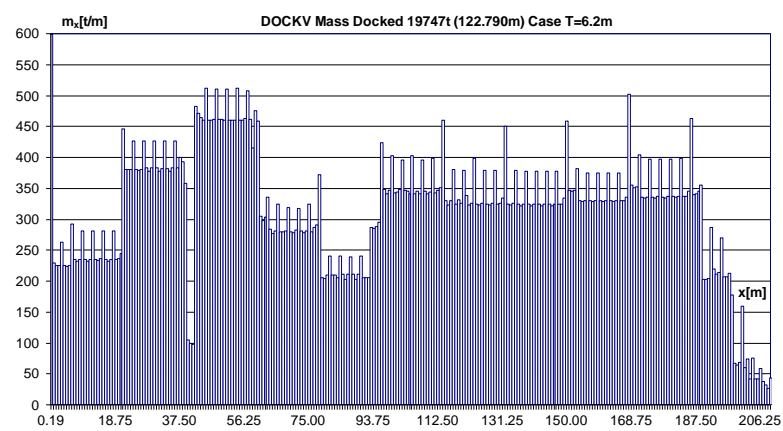

Fig. 9a. DOCKV D19747t ( \& ball), mass disitribution, shipyard docking case, $T_{m}=6.2 \mathrm{~m}$ [2]

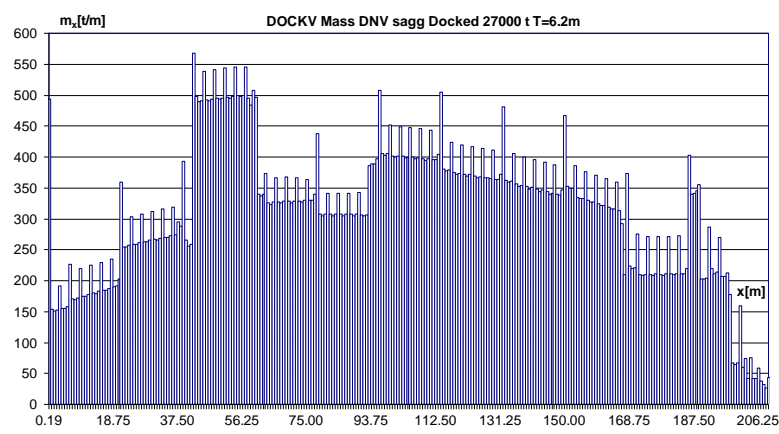

Fig. 9c. DOCKV D27000t (\&ball) sagg-mass, with sagging docking mass distribution, $T_{m}=6.2 \mathrm{~m} \mathrm{[2]}$

considered only for $\mu=0 \div 90^{\circ}$, with $\delta \mu=15^{\circ}$ step. The global strength analysis by equivalent beam model, under oblique waves, of the NWT-Dock60 and CWTDock60 versions, using P_QSWD [6] program, with modules 5.1-5.4 from Fig. 1, lead to the global strength and free board criteria check for all four loading cases from Tables 3a-d (CWT) and Tables 4ad (NWT).

Table 3a. Global strength and free board criteria, NWT Dock60 light displacement case

\begin{tabular}{|c|c|c|c|c|c|c|c|c|c|c|c|c|c|c|c|}
\hline $\begin{array}{l}\text { NWT } \\
\text { light }\end{array}$ & $\mu\left[{ }^{\circ}\right]$ & 0 & $\begin{array}{l}\max / \\
\operatorname{adm} \\
\end{array}$ & 15 & $\begin{array}{c}\max / \\
\operatorname{adm}\end{array}$ & 30 & $\begin{array}{l}\max / \\
\operatorname{adm}\end{array}$ & 45 & $\begin{array}{l}\max / \\
\text { adm }\end{array}$ & 60 & $\begin{array}{l}\max / \\
\text { adm }\end{array}$ & 75 & $\begin{array}{l}\max / \\
\mathrm{adm}\end{array}$ & 9 & $\begin{array}{c}\max / \\
\text { adm }\end{array}$ \\
\hline$h_{\text {wlim }}[\mathrm{m}]$ & wave & \multicolumn{2}{|c|}{0.640} & \multicolumn{2}{|c|}{0.666} & \multicolumn{2}{|c|}{0.778} & \multicolumn{2}{|c|}{1.278} & \multicolumn{2}{|c|}{1.800} & \multicolumn{2}{|c|}{1.800} & \multicolumn{2}{|c|}{1.800} \\
\hline Free & hogg & 880 & $>1$ & 867 & $>1$ & .811 & $>1$ & .561 & $>1$ & 300 & 1.00 & .300 & 1.00 & .300 & 1.00 \\
\hline ard & sagg & 880 & $>1$ & 0.867 & $>1$ & 0.811 & $>1$ & .561 & $>1$ & 0.300 & 00 & 0.300 & & 0.300 & 00 \\
\hline VBM & hogg & $.27 \mathrm{E}+4$ & 1.00 & $2.27 \mathrm{E}+4$ & 1.00 & $2.27 \mathrm{E}+4$ & 1.00 & $27 \mathrm{E}+4$ & 1.00 & $1.01 \mathrm{E}+4$ & 0.45 & $5.43 E+3$ & 0.28 & 0 & 0 \\
\hline $\mathrm{kNm}$ & sagg & $99 \mathrm{E}+2$ & 0.01 & $2.99 \mathrm{E}+2$ & 0.01 & $2.96 \mathrm{E}+2$ & \begin{tabular}{|l|}
0.01 \\
\end{tabular} & $3 \mathrm{E}+2$ & 0.01 & $1.58 \mathrm{E}+4$ & 0.69 & $\mathrm{E}+4$ & 0.95 & 0 & 0 \\
\hline$\overline{\mathrm{VSF}}$ & hogg & $3 \mathrm{E}+3$ & 0.42 & $1.33 \mathrm{E}+3$ & 0.42 & $1.33 \mathrm{E}+3$ & 0.42 & $3 E+3$ & 0. & $5.61 E+2$ & 8 & $5 \mathrm{E}+2$ & 0 & & $\overline{0}$ \\
\hline$[\mathrm{kN}]$ & sagg & $.29 \mathrm{E}+2$ & 0.04 & $1.29 \mathrm{E}+2$ & 0.04 & $1.29 \mathrm{E}+2$ & \begin{tabular}{|l|}
0.04 \\
\end{tabular} & $.30 \mathrm{E}+2$ & 0.04 & $1 \mathrm{E}+2$ & 0.28 & $56 \mathrm{E}+3$ & 0.49 & ( & \\
\hline $\mathrm{BM}$ & $g \mathrm{~g}$ & 0 & 0 & $6.67 \mathrm{E}+1$ & 0.02 & $1.60 \mathrm{E}+2$ & 0.04 & $4.00 \mathrm{E}+2$ & 0.09 & $E+2$ & 0.13 & $7 \mathrm{E}+2$ & 0.07 & 0 & 0 \\
\hline Jm] & 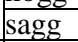 & 0 & 0 & & & +2 & & +2 & 87 & & 15 & $0 \mathrm{E}+2$ & 1 & & 0 \\
\hline $\mathrm{HSF}$ & $\operatorname{tg}$ & 0 & 0 & -0 & 0.04 & $E+1$ & 0.10 & +1 & 0.26 & $E+1$ & 0.29 & $05 \mathrm{E}+1$ & 0.14 & 0 & 0 \\
\hline$[\mathrm{kN}]$ & sagg & 0 & 0 & $4.84 \mathrm{E}+0$ & 0.02 & $1.16 \mathrm{E}+1$ & \begin{tabular}{|l|}
0.05 \\
\end{tabular} & $2.91 \mathrm{E}+1$ & 0.14 & \begin{tabular}{|l|}
$8.19 \mathrm{E}+1$ \\
\end{tabular} & 0.39 & $6.07 \mathrm{E}+1$ & 0.29 & 0 & 0 \\
\hline & hogg & 0 & 0 & 1.72 & 0.07 & $4.12 \mathrm{E}+3$ & \begin{tabular}{|l|}
0.17 \\
\end{tabular} & $1.03 \mathrm{E}+4$ & 0.42 & $1.61 \mathrm{E}+4$ & 0.66 & $1.10 \mathrm{E}+4$ & 0.45 & $.59 \mathrm{E}+4$ & 0.7 \\
\hline $\mathrm{Nm}$ & sagg & 0 & 0 & $1.72 \mathrm{E}+3$ & 0.07 & $4.13 \mathrm{E}+3$ & 0.17 & $1.03 \mathrm{E}+4$ & 0.42 & $1.61 \mathrm{E}+4$ & 0.66 & $1.10 \mathrm{E}+4$ & 0.45 & $4.98 \mathrm{E}+3$ & 0.2 \\
\hline
\end{tabular}


Table 3b. Global strength and free board criteria, NWT Dock60 ship 1 displacement case

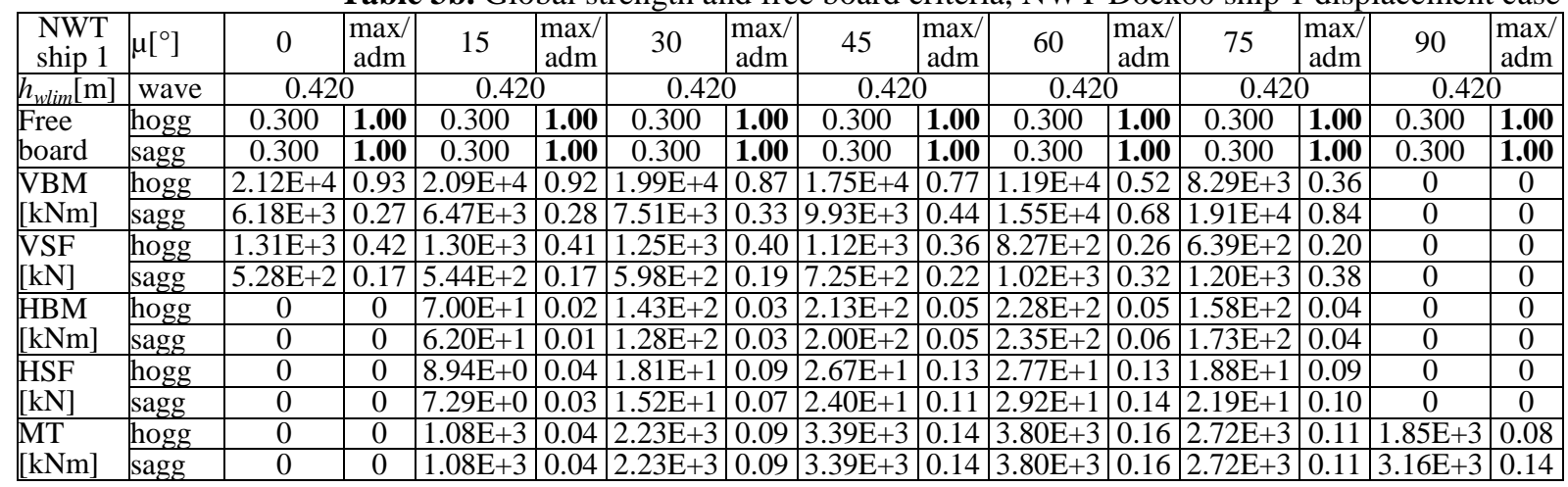

Table 3c. Global strength and free board criteria, NWT Dock60 ship 2 displacement case

\begin{tabular}{|c|c|c|c|c|c|c|c|c|c|c|c|c|c|c|c|}
\hline $\begin{array}{l}\text { NWT } \\
\text { ship 2 }\end{array}$ & & 0 & $\begin{array}{l}\max / \\
\text { adm }\end{array}$ & 15 & $\begin{array}{l}\max / \\
\operatorname{adm}\end{array}$ & 30 & $\begin{array}{l}\max / \\
\operatorname{adm}\end{array}$ & 45 & $\begin{array}{c}\max / \\
\operatorname{adm}\end{array}$ & 60 & $\begin{array}{l}\max / \\
\operatorname{adm}\end{array}$ & 75 & $\begin{array}{l}\max / \\
\operatorname{adm}\end{array}$ & 90 & $\begin{array}{l}\max / \\
\operatorname{adm}\end{array}$ \\
\hline$h_{\text {wlim }}[\mathrm{m}]$ & wave & \multicolumn{2}{|c|}{0.420} & \multicolumn{2}{|c|}{0.420} & \multicolumn{2}{|c|}{0.420} & \multicolumn{2}{|c|}{0.420} & \multicolumn{2}{|c|}{0.420} & \multicolumn{2}{|c|}{0.420} & \multicolumn{2}{|c|}{0.420} \\
\hline Free & hogg & .300 & 1.00 & 0.300 & 1.00 & 0.300 & 1.00 & .300 & 0.300 & 1.00 & 0.300 & 1.00 & 0.300 & 1.00 & 0.30 \\
\hline oard & sagg & 0.300 & 1.00 & 0.300 & 1.00 & 0.300 & 1.00 & 0.300 & 0.300 & 1.00 & 0.300 & 1.00 & 0.300 & 1.00 & 0.30 \\
\hline$\overline{B M}$ & hogg & $8+4$ & 0.61 & $.35 E+4$ & & & & & 0.44 & $.50 \mathrm{E}+3$ & & $.36 \mathrm{E}$ & & 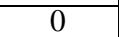 & \\
\hline$[\mathrm{kNm}]$ & sagg & & & & & & & & & & & & & & \\
\hline & hogg & +2 & 31 & & 0.31 & -2 & 0.29 & +2 & 25 & +2 & 6 & $3.14 \mathrm{E}+2$ & 0 & 0 & 0 \\
\hline & & $E+2$ & 0.08 & & & & & & & & & & & 0 & 0 \\
\hline $3 \mathrm{M}$ & 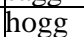 & 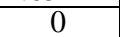 & $\pi$ & & 2 & & $f$ & & 55 & & & +2 & 4 & & \\
\hline$[\mathrm{kNm}]$ & sagg & 0 & 0 & $6.20 \mathrm{E}+1$ & 0.01 & $E+2$ & 0.03 & $\mathrm{E}+2$ & 0.05 & $5 \mathrm{E}+2$ & 6 & $3 \mathrm{E}+2$ & .04 & 0 & \\
\hline & $\mathrm{gg}$ & 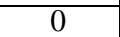 & & & 0.04 & & 0.09 & & 3 & & & $\mathrm{E}+1$ & 09 & $\Omega$ & 0 \\
\hline & & & 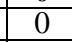 & & & & & & & & & & .10 & 0 & \\
\hline & & 0 & ( & & 0 & $23 E+3$ & 0. & -3 & & +3 & & +3 & 11 & $1.85 \mathrm{E}$ & 0.0 \\
\hline $\mathrm{kNm}]$ & sagg & 0 & 0 & $1.08 \mathrm{E}+3$ & 0.04 & $2.23 \mathrm{E}+3$ & \begin{tabular}{|l|}
0.09 \\
\end{tabular} & $3.39 \mathrm{E}+3$ & \begin{tabular}{|l|} 
\\
\end{tabular} & $3.80 \mathrm{E}+3$ & 0.16 & $2.72 \mathrm{E}+3$ & \begin{tabular}{|l|}
0.11 \\
\end{tabular} & $3.16 \mathrm{E}+3$ & 0.14 \\
\hline
\end{tabular}

Table 3d. Global strength and free board criteria, NWT Dock60 ship 3 displacement case

\begin{tabular}{|c|c|c|c|c|c|c|c|c|c|c|c|c|c|c|c|}
\hline $\begin{array}{l}\text { NWT } \\
\text { ship3 }\end{array}$ & $\mu\left[^{\circ}\right]$ & 0 & $\begin{array}{c}\max / \\
\text { adm }\end{array}$ & 15 & $\begin{array}{c}\max / \\
\mathrm{adm}\end{array}$ & 30 & $\begin{array}{c}\max / \\
\operatorname{adm}\end{array}$ & 45 & $\begin{array}{c}\max / \\
\text { adm }\end{array}$ & 60 & \begin{tabular}{|l|}
$\max /$ \\
$\operatorname{adm}$
\end{tabular} & 75 & $\begin{array}{c}\mathrm{Tax} / \\
\mathrm{adm}\end{array}$ & 90 & $\begin{array}{l}\max / \\
\mathrm{adm}\end{array}$ \\
\hline$h_{\text {wlim }}[\mathrm{m}]$ & wave & \multicolumn{2}{|c|}{0.261} & \multicolumn{2}{|c|}{0.272} & \multicolumn{2}{|c|}{0.318} & \multicolumn{2}{|c|}{0.420} & \multicolumn{2}{|c|}{0.420} & \multicolumn{2}{|c|}{0.420} & \multicolumn{2}{|c|}{0.420} \\
\hline Free & hogg & 0.379 & 1.26 & 0.374 & 1.24 & 0.351 & 1.17 & 0.300 & 1.00 & 0.300 & \begin{tabular}{|l|}
1.00 \\
\end{tabular} & .300 & 1.00 & 0.300 & 1.00 \\
\hline board & sagg & 0.379 & 1.26 & 0.374 & 1.24 & 0.351 & 1.17 & 0300 & & & & 0.300 & 0 & 0.300 & 1.00 \\
\hline /BM & hogg & $27 \mathrm{E}+4$ & 1.00 & $.27 \mathrm{E}+4$ & 1.00 & $27 \mathrm{E}+$ & & & & & & & & & \\
\hline$[\mathrm{kNm}]$ & sagg & $34 \mathrm{E}+4$ & 0.59 & $\mathrm{E}+4$ & 0.59 & $1.34 \mathrm{E}+4$ & 0.59 & & 0.63 & 1.9 & \begin{tabular}{|l|}
0.86 \\
\end{tabular} & +4 & 96 & & 0 \\
\hline & hogg & $7 \mathrm{E}+3$ & 0.44 & $\mathrm{E}+3$ & 0.46 & $1.44 \mathrm{E}+3$ & 0.46 & & 0.44 & & 0.35 & $18 \mathrm{E}+2$ & 29 & 0 & 0 \\
\hline$[\mathrm{kN}]$ & sagg & $56 \mathrm{E}+2$ & 0.30 & 2 & 0.30 & $9.55 \mathrm{E}$ & 0.30 & & 2 & & 0.41 & $3 \mathrm{E}+3$ & 47 & & 0 \\
\hline $\mathrm{HBM}$ & hogg & 0 & 0 & $4.44 \mathrm{E}+1$ & 0.01 & $1.07 \mathrm{E}+2$ & 0.03 & $2.13 \mathrm{E}+2$ & 0.05 & +2 & .05 & $8 \mathrm{E}+2$ & .04 & & 0 \\
\hline$[\mathrm{kNm}]$ & gg & 0 & 0 & $4.10 \mathrm{E}+1$ & 0.01 & $9.85 \mathrm{E}+1$ & 0.02 & $2.00 \mathrm{E}+2$ & 0.05 & $\mathrm{E}+2$ & 0.06 & $1.73 \mathrm{E}+2$ & 0.04 & 0 & 0 \\
\hline SE & $\mathrm{gg}$ & 0 & 0 & & 0.03 & & 0.06 & +1 & 0.13 & & 0.13 & $E+1$ & 0.09 & & 0 \\
\hline $\mathrm{VI}$ & & 0 & 0 & 0 & 0.02 & & 0.06 & $8+1$ & & & \begin{tabular}{|l|}
0.14 \\
\end{tabular} & $\mathrm{E}+1$ & 0.10 & 0 & 0 \\
\hline IVI & hogg & 0 & 0 & $7.02 \mathrm{E}+2$ & 0.03 & $1.69 \mathrm{E}+3$ & 0.07 & $3.39 \mathrm{E}+3$ & 0.14 & $3.80 \mathrm{E}+3$ & 0.16 & $2.72 \mathrm{E}+3$ & 0.11 & $1.85 \mathrm{E}+3$ & 0.08 \\
\hline$[\mathrm{kNm}]$ & sagg & 0 & 0 & $7.02 \mathrm{E}+2$ & 0.03 & $1.69 \mathrm{E}+3$ & 0.07 & $3.39 \mathrm{E}+3$ & 0.14 & $3.80 \mathrm{E}+3$ & $\mid 0.16$ & $2.72 \mathrm{E}+3$ & 0.11 & $3.16 \mathrm{E}+3$ & 0.14 \\
\hline
\end{tabular}

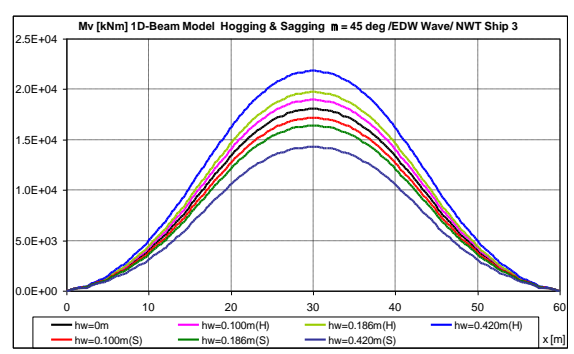

Fig. 10a. VBM, ship3, $\mu=45^{\circ}$, NWT

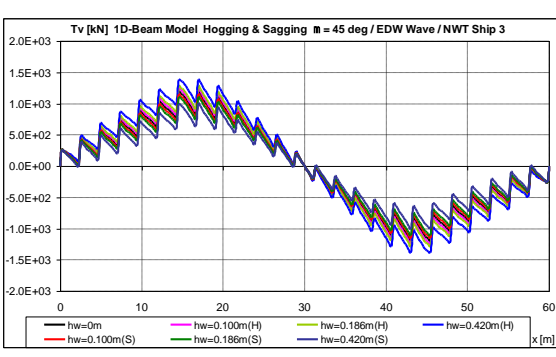

Fig. 10b. VSF, ship $3, \mu=45^{\circ}$, NWT

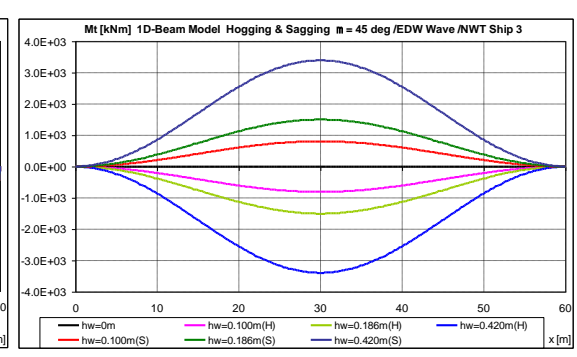

Fig. 10c. MT, ship3, $\mu=45^{\circ}$, NWT

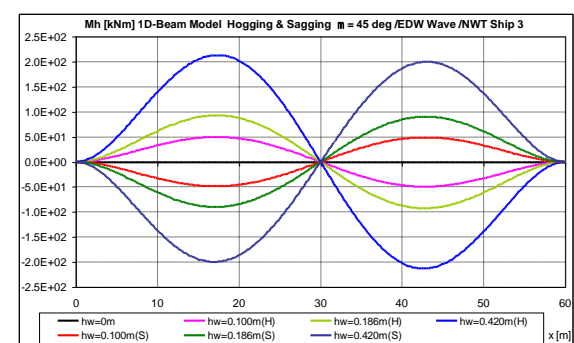

Fig. 10d. HBM, ship3, $\mu=45^{\circ}$, NWT

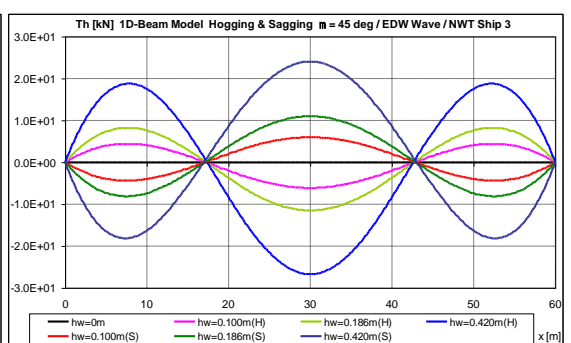

Fig. 10e. HSF, ship $3, \mu=45^{\circ}$, NWT 
Table 4a. Global strength and free board criteria, CWT Dock60 light displacement case

\begin{tabular}{|c|c|c|c|c|c|c|c|c|c|c|c|c|c|c|c|}
\hline $\begin{array}{l}\text { CWT } \\
\text { light }\end{array}$ & $\mu\left[{ }^{\circ}\right]$ & 0 & $\begin{array}{l}\max / \\
\operatorname{adm}\end{array}$ & 15 & $\begin{array}{l}\max / \\
\operatorname{adm}\end{array}$ & 30 & $\begin{array}{l}\max / \\
\operatorname{adm}\end{array}$ & 45 & $\begin{array}{l}\max / \\
\operatorname{adm}\end{array}$ & 60 & $\begin{array}{l}\max / \\
\operatorname{adm}\end{array}$ & 75 & $\begin{array}{l}\max / \\
\operatorname{adm}\end{array}$ & 90 & $\begin{array}{c}\max / \\
\operatorname{adm}\end{array}$ \\
\hline$h_{w \lim }[\mathrm{m}]$ & wave & \multicolumn{2}{|c|}{1.930} & \multicolumn{2}{|c|}{1.930} & \multicolumn{2}{|c|}{1.930} & \multicolumn{2}{|c|}{1.930} & \multicolumn{2}{|c|}{1.930} & \multicolumn{2}{|c|}{1.930} & \multicolumn{2}{|c|}{1.930} \\
\hline Free & hogg & 0.075 & 1.00 & 0.075 & 1.00 & .075 & 1.00 & 0.075 & 1.00 & 0.075 & 1.00 & .075 & 1.00 & 0.075 & 1.00 \\
\hline board & sagg & 0.075 & 1.00 & 0.075 & 1.00 & 0.075 & 1.00 & 0.075 & 1.00 & 0.075 & 1.00 & 0.075 & 1.00 & 0.075 & 1.00 \\
\hline VBM & hogg & $.40 \mathrm{E}+4$ & 0.79 & $4.27 \mathrm{E}+4$ & 0.77 & $3.82 \mathrm{E}+4$ & 0.69 & $2.81 \mathrm{E}+4$ & 0.51 & $1.97 \mathrm{E}+3$ & 0.04 & $1.53 \mathrm{E}+4$ & 0.28 & , & 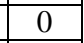 \\
\hline$[\mathrm{kNm}]$ & sagg & & 0.45 & & 0.43 & +4 & 0.35 & & 0.14 & & 0.26 & +4 & 0.62 & & 0 \\
\hline VSF & hogg & +3 & 77 & +3 & 0.75 & +3 & 0.67 & & 0.50 & +2 & 0.06 & $\mathrm{E}+2$ & 0.25 & & 0 \\
\hline$[\mathrm{kN}]$ & sagg & $28 \mathrm{E}+3$ & 0.41 & $\overline{E+3}$ & 0.39 & $\bar{E}+2$ & 0.31 & +2 & 13 & +2 & 0.29 & $E+3$ & 0. & & 0 \\
\hline $\mathrm{HBM}$ & hogg & 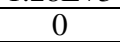 & 0 & $2.87 \mathrm{E}+2$ & 0.06 & $5.63 \mathrm{E}+2$ & 0.11 & +2 & 0.15 & +2 & 0.15 & $3.54 \mathrm{E}+2$ & 0.07 & & 0 \\
\hline$[\mathrm{kNm}]$ & sagg & ( & 0 & $27 \mathrm{E}+2$ & 0.02 & $2.77 \mathrm{E}+2$ & 0.05 & $4.94 \mathrm{E}+2$ & 0.10 & $4.94 \mathrm{E}+2$ & 0.10 & $6.87 \mathrm{E}+2$ & 0.13 & & 0 \\
\hline ISF & hogg & ( & 0 & $15 \mathrm{E}+1$ & 0.16 & $8.01 \mathrm{E}+1$ & 0.32 & $1.04 \mathrm{E}+2$ & 0.41 & $6.94 \mathrm{E}+1$ & 0.27 & $3.90 \mathrm{E}+1$ & 0.15 & & 0 \\
\hline$[\mathrm{kN}]$ & sagg & ( & 0 & $52 \mathrm{E}+1$ & 0.06 & $3.16 \mathrm{E}+1$ & 0.12 & $5.05 \mathrm{E}+1$ & 0.20 & $9.87 \mathrm{E}+1$ & 0.39 & $9.63 \mathrm{E}+1$ & 0.38 & 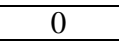 & 0 \\
\hline $\mathrm{T}$ & hogg & 0 & 0 & $5.06 \mathrm{E}+3$ & 0.21 & $1.04 \mathrm{E}+4$ & 0.43 & $1.58 \mathrm{E}+4$ & 0.65 & $1.76 \mathrm{E}+4$ & 0.72 & $1.26 \mathrm{E}+4$ & 0.51 & $.15 \mathrm{E}+4$ & 0.39 \\
\hline $\mathrm{kNm}]$ & sagg & 0 & 0 & $5.00 \mathrm{E}+3$ & 0.20 & $1.03 \mathrm{E}+4$ & 0.42 & $1.57 \mathrm{E}+4$ & 0.64 & $1.77 \mathrm{E}+4$ & 0.72 & $1.27 \mathrm{E}+4$ & 0.52 & $1.46 \mathrm{E}+3$ & 0.03 \\
\hline
\end{tabular}

Table 4b. Global strength and free board criteria, CWT Dock60 ship 1 displacement case

\begin{tabular}{|c|c|c|c|c|c|c|c|c|c|c|c|c|c|c|c|}
\hline $\begin{array}{l}\text { CWT } \\
\text { ship1 } \\
\end{array}$ & $\mu\left[^{\circ}\right]$ & 0 & $\begin{array}{l}\max / \\
\operatorname{adm}\end{array}$ & 15 & $\begin{array}{l}\max / \\
\text { adm }\end{array}$ & 30 & $\begin{array}{l}\max / \\
\text { adm }\end{array}$ & 45 & $\begin{array}{l}\max / \\
\operatorname{adm}\end{array}$ & 60 & $\begin{array}{l}\max / \\
\text { adm }\end{array}$ & 75 & $\begin{array}{l}\max / \\
\operatorname{adm}\end{array}$ & 90 & $\begin{array}{c}\max / \\
\operatorname{adm}\end{array}$ \\
\hline$h_{w l i m}[\mathrm{~m}]$ & wave & \multicolumn{2}{|c|}{0.550} & \multicolumn{2}{|c|}{0.550} & \multicolumn{2}{|c|}{0.550} & \multicolumn{2}{|c|}{0.550} & \multicolumn{2}{|c|}{0.550} & \multicolumn{2}{|c|}{0.550} & \multicolumn{2}{|c|}{0.550} \\
\hline Free & hogg & 0.075 & 1.00 & 075 & 1.00 & 075 & 1.00 & 075 & 1.00 & 0.075 & 1.00 & .075 & 1.00 & 0.075 & \begin{tabular}{|l|}
1.00 \\
\end{tabular} \\
\hline board & sagg & 0.075 & 1.00 & 0.075 & 1.00 & 075 & 1.00 & 0.075 & 1.00 & 075 & 1.00 & .075 & 0 & 0.075 & 1.00 \\
\hline VBM & hogg & $17 \mathrm{E}+4$ & 0.39 & $2.13 E+4$ & 0.38 & $2.00 \mathrm{E}+4$ & 0.36 & $1.68 \mathrm{E}+4$ & 0.30 & $3 \mathrm{E}+3$ & 0.17 & +3 & 0.09 & & 0 \\
\hline$[\mathrm{kNm}]$ & sagg & $26 \mathrm{E}+3$ & 0.04 & $2.56 \mathrm{E}+3$ & 0.05 & $3.79 \mathrm{E}+3$ & 0.07 & $6.96 \mathrm{E}+3$ & 0.13 & $2 \mathrm{E}+4$ & 0.26 & $\mathrm{E}+4$ & .34 & & 0 \\
\hline VSF & hogg & $33 \mathrm{E}+3$ & 0.42 & $1.31 \mathrm{E}+3$ & 0.42 & $1.24 \mathrm{E}+3$ & 0.39 & $1.07 \mathrm{E}+3$ & 0.34 & $6.92 \mathrm{E}+2$ & 0.22 & $5 \mathrm{E}+2$ & 0.14 & & 0 \\
\hline$[\mathrm{kN}]$ & sagg & $3.71 \mathrm{E}+2$ & 0.12 & $3.71 \mathrm{E}+2$ & 0.12 & $3.92 \mathrm{E}+2$ & 0.12 & $5.58 \mathrm{E}+2$ & 0.18 & $9.39 \mathrm{E}+2$ & 0.30 & $9 \mathrm{E}+3$ & 0.38 & ( & 0 \\
\hline $\mathrm{HBM}$ & hogg & 0 & 0 & $1.03 \mathrm{E}+2$ & 0.02 & $2.09 \mathrm{E}+2$ & 0.04 & $3.10 \mathrm{E}+2$ & 0.06 & $3.29 \mathrm{E}+2$ & 0.06 & $2.27 \mathrm{E}+2$ & 0.04 & ( & 0 \\
\hline$[\mathrm{kNm}]$ & sagg & 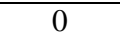 & 0 & $8.89 \mathrm{E}+1$ & 0.02 & $1.84 \mathrm{E}+2$ & 0.04 & $2.88 \mathrm{E}+2$ & 0.06 & $3.41 \mathrm{E}+2$ & 0.07 & $2.53 \mathrm{E}+2$ & 0.05 & 0 & 0 \\
\hline HSF & hogg & 0 & 0 & $1.32 \mathrm{E}+1$ & 0.05 & $2.66 \mathrm{E}+1$ & 0.11 & $3.91 \mathrm{E}+1$ & 0.15 & $4.00 \mathrm{E}+1$ & 0.16 & $2.68 \mathrm{E}+1$ & 0.11 & 0 & 0 \\
\hline$[\mathrm{kN}]$ & sagg & 0 & 0 & $1.04 \mathrm{E}+1$ & 0.04 & $2.17 \mathrm{E}+1$ & 0.09 & $3.45 \mathrm{E}+1$ & 0.14 & $4.25 \mathrm{E}+1$ & 0.17 & $3.21 \mathrm{E}+1$ & 0.13 & 0 & 0 \\
\hline MT & hogg & 0 & 0 & $1.45 \mathrm{E}+3$ & 0.06 & $2.97 \mathrm{E}+3$ & 0.12 & $4.53 \mathrm{E}+3$ & 0.19 & $5.07 \mathrm{E}+3$ & 0.21 & $3.62 \mathrm{E}+3$ & 0.15 & $23 E+3$ & 0.13 \\
\hline$[\mathrm{kNm}]$ & sagg & 0 & 0 & $1.44 \mathrm{E}+3$ & 0.06 & $2.96 \mathrm{E}+3$ & 0.12 & $4.52 \mathrm{E}+3$ & 0.18 & $5.07 \mathrm{E}+3$ & 0.21 & $3.63 \mathrm{E}+3$ & 0.15 & $9.11 \mathrm{E}+2$ & 0.02 \\
\hline
\end{tabular}

Table 4c. Global strength and free board criteria, CWT Dock60 ship 2 displacement case

\begin{tabular}{|c|c|c|c|c|c|c|c|c|c|c|c|c|c|c|c|}
\hline $\begin{array}{l}\text { CWT } \\
\text { ship2 }\end{array}$ & $\mu\left[^{\circ}\right]$ & 0 & $\mathrm{me}$ & 15 & $\begin{array}{l}\max / \\
\text { adm }\end{array}$ & 30 & $\begin{array}{l}\max / \\
\operatorname{adm}\end{array}$ & 45 & $\begin{array}{l}\max / \\
\operatorname{adm}\end{array}$ & 0 & $\begin{array}{l}\max / \\
\operatorname{adm}\end{array}$ & 75 & $\begin{array}{l}\max / \\
\operatorname{adm}\end{array}$ & 90 & \begin{tabular}{|c|}
$\max /$ \\
$\operatorname{adm}$
\end{tabular} \\
\hline wlim $[\mathrm{m}]$ & av & \multicolumn{2}{|c|}{0.550} & \multicolumn{2}{|c|}{0.550} & \multicolumn{2}{|c|}{0.550} & \multicolumn{2}{|c|}{0.550} & \multicolumn{2}{|c|}{0.550} & \multicolumn{2}{|c|}{0.550} & \multicolumn{2}{|c|}{0.550} \\
\hline & hogg & 0.075 & 1.00 & 0.075 & 1.00 & 0.075 & 1.00 & 0.075 & 1.00 & 0.075 & 1.00 & 0.075 & 1.00 & 0.075 & \begin{tabular}{|l|}
1.00 \\
\end{tabular} \\
\hline oard & sagg & 0.075 & 1.00 & 0.075 & 1.00 & 0.075 & 1.00 & 0.075 & 1.00 & 0.075 & 1.00 & 0.075 & 1.00 & 0.075 & 1.00 \\
\hline VBM & hogg & $3 \mathrm{E}+4$ & 0.26 & $.39 \mathrm{E}+4$ & 0.25 & $26 \mathrm{E}+4$ & 0.23 & $9.41 \mathrm{E}+3$ & 0.17 & $13 \mathrm{E}+3$ & 0.04 & $2.68 \mathrm{E}+3$ & 0.05 & & 0 \\
\hline$[\mathrm{kNm}]$ & sagg & $3 E+3$ & 10 & $5.05 \mathrm{E}+3$ & 0.09 & $\mathrm{E}+3$ & 0.07 & & 0.01 & +3 & 0.12 & & 0.2 & & \\
\hline VSF & hogg & $1 \mathrm{E}+3$ & .32 & $9.85 \mathrm{E}+2$ & 0.31 & $9.14 \mathrm{E}+2$ & 0.29 & 7.4 & 0.24 & +2 & 0.12 & & ti & & 0 \\
\hline$[\mathrm{kN}]$ & sagg & $32 \mathrm{E}+2$ & 0.14 & $\mathrm{E}+2$ & 0.13 & $\mathrm{E}+2$ & .11 & -2 & 0.07 & +2 & 0.20 & +2 & 0.27 & & 0 \\
\hline $\mathrm{HBM}$ & hogg & 0 & 0 & 2 & 0.02 & $E+2$ & .04 & -2 & 0.06 & & 0.06 & 2 & 0.04 & & 0 \\
\hline$[\mathrm{kNm}]$ & sagg & & ( & & 0.02 & $E+2$ & 0.04 & & 0.06 & $E+2$ & 0.07 & & 0.05 & & 0 \\
\hline $\mathrm{HSF}$ & hogg & ( & 0 & & 0.05 & +1 & 0.11 & & 0.15 & $E+1$ & 0.16 & & 0.11 & & 0 \\
\hline$[\mathrm{kN}]$ & sagg & & c & & 0.04 & $E+1$ & 0.09 & & 0.14 & $\mathrm{E}+1$ & 0.17 & & 0.13 & 0 & 0 \\
\hline IT & hogg & ( & c & $1.45 \mathrm{E}+3$ & 0.06 & $2.97 \mathrm{E}+3$ & 0.12 & & 0.19 & $E+3$ & 0.21 & +3 & 0.15 & $7.23 \mathrm{E}+3$ & 0.13 \\
\hline $\mathrm{kNm}]$ & sagg & 0 & 0 & $1.44 \mathrm{E}+3$ & 0.06 & $2.96 \mathrm{E}+3$ & 0.12 & $4.52 \mathrm{E}+3$ & 0.18 & $5.07 \mathrm{E}+3$ & 0.21 & $3.63 \mathrm{E}+3$ & 0.15 & $9.11 \mathrm{E}+2$ & 0.0 \\
\hline
\end{tabular}

Table 4d. Global strength and free board criteria, CWT Dock60 ship 3 displacement case

\begin{tabular}{|c|c|c|c|c|c|c|c|c|c|c|c|c|c|c|c|}
\hline $\begin{array}{l}\text { CWT } \\
\text { ship3 }\end{array}$ & $\left.{ }^{\circ}\right]$ & 0 & $\begin{array}{l}\text { max/ } \\
\text { adm }\end{array}$ & 15 & $\begin{array}{c}\max / \\
\text { adm }\end{array}$ & 30 & $\begin{array}{l}\max / \\
\operatorname{adm}\end{array}$ & 45 & $\begin{array}{l}\max / \\
\text { adm }\end{array}$ & 60 & $\begin{array}{l}\max / \\
\text { adm }\end{array}$ & 75 & $\begin{array}{l}\text { max/ } \\
\text { adm }\end{array}$ & 9 & $\begin{array}{c}\max / \\
\text { adm }\end{array}$ \\
\hline$h_{\text {wlim }}[\mathrm{m}]$ & wave & \multicolumn{2}{|c|}{0.550} & \multicolumn{2}{|c|}{0.550} & \multicolumn{2}{|c|}{0.550} & \multicolumn{2}{|c|}{0.550} & \multicolumn{2}{|c|}{0.550} & \multicolumn{2}{|c|}{0.550} & \multicolumn{2}{|c|}{0.550} \\
\hline Free & hogg & 075 & 1.00 & 075 & 1.00 & 075 & 1.00 & 075 & 1.00 & 075 & 00 & .075 & 1.00 & .075 & 1.00 \\
\hline $\operatorname{ard}$ & sagg & & & 075 & & 075 & & 075 & & & & & & .075 & \\
\hline $\mathrm{BM}$ & hogg & $61 \mathrm{E}+4$ & 0.47 & $2.57 \mathrm{E}+4$ & 0.46 & $2.44 \mathrm{E}+4$ & 0.44 & $2 E+4$ & 0.38 & $1.39 \mathrm{E}+4$ & 0.25 & $3 E+3$ & 0.17 & 0 & 0 \\
\hline [kNm] & sagg & & 12 & $E+3$ & 0.12 & & & $\mathrm{E}+4$ & 0.20 & & 0.33 & +4 & 0.42 & 0 & 0 \\
\hline $\mathrm{ISF}$ & hogg & +3 & 51 & & 050 & +3 & & & 043 & & & & 0.2 & & \\
\hline $\mathrm{kN}]$ & sagg & $78 \mathrm{E}+2$ & \begin{tabular}{|l|}
0.18 \\
\end{tabular} & $5.98 \mathrm{E}+2$ & 0.19 & $67 \mathrm{E}+2$ & 0. & +2 & 26 & +3 & 0.39 & +3 & + & & 0 \\
\hline HBM & hogg & 0 & 0 & $1.03 \mathrm{E}+2$ & 0.02 & $2.09 \mathrm{E}+2$ & 0.04 & $0 \mathrm{E}+2$ & 0. & $E+2$ & 0.06 & $7 \mathrm{E}+2$ & 0.04 & 0 & 0 \\
\hline $\mathrm{Jm}]$ & SSa & 0 & 0 & & & & 0. & & 80 & & 0.07 & & 0.05 & & 0 \\
\hline & & 0 & 0 & 1 & & +1 & 0 & +1 & & $\mathrm{E}+1$ & 0.16 & $E+1$ & 0. & 0 & 0 \\
\hline$[\mathrm{kN}]$ & sagg & 0 & 0 & $1.04 \mathrm{E}+1$ & 0.04 & $2.17 \mathrm{E}+1$ & 0.09 & $3.45 \mathrm{E}+1$ & 0.14 & $4.25 \mathrm{E}+1$ & 0.17 & $3.21 \mathrm{E}+1$ & 0.13 & 0 & 0 \\
\hline & hogg & 0 & 0 & $1.45 \mathrm{E}+3$ & 0.06 & $2.97 \mathrm{E}+3$ & 0.12 & $4.53 \mathrm{E}+3$ & 0.19 & $5.07 \mathrm{E}+3$ & 0.21 & $3.62 \mathrm{E}+3$ & 0.15 & $23 \mathrm{E}+3$ & 0.13 \\
\hline $\mathrm{Nm}$ & sagg & 0 & 0 & $1.44 \mathrm{E}+3$ & 0.06 & $2.96 \mathrm{E}+3$ & 0.12 & $4.52 \mathrm{E}+3$ & 0.18 & $5.07 \mathrm{E}+3$ & 0.21 & $3.63 \mathrm{E}+3$ & 0.15 & $.11 \mathrm{E}+2$ & 0.0 \\
\hline
\end{tabular}




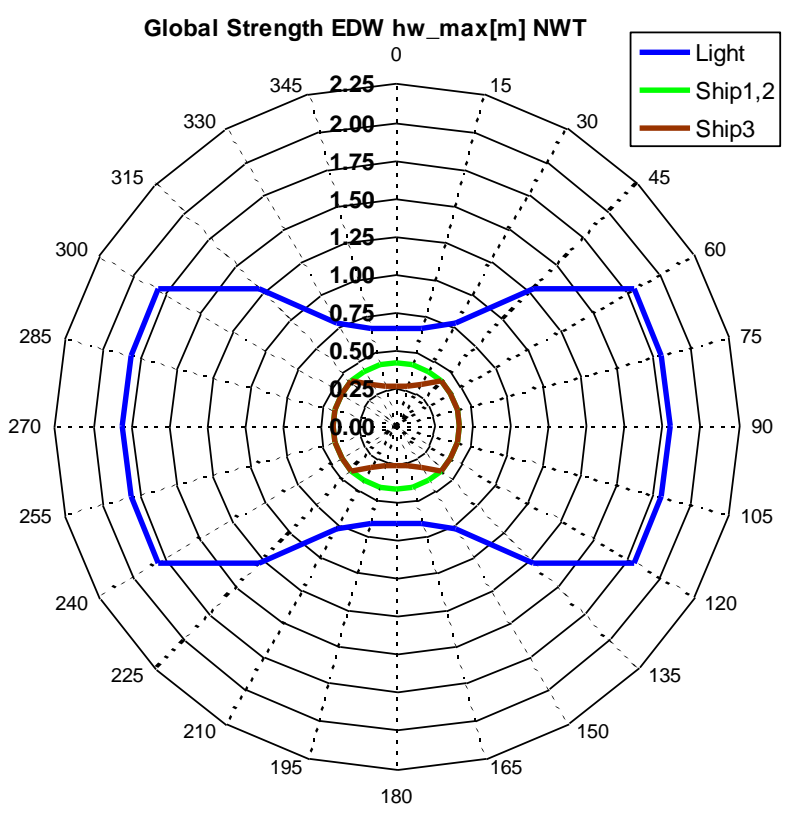

Fig. 11a. NWT-Dock60 polar diagram of EDW wave height limit $h_{\text {wlimit }}$ all four loading cases, beam model

Figures 10a-e present the VBM, VSF, HBM, HSF, MT diagrams at $\mu=45^{\circ}$, for NWT-Dock60 ship 3 case

Figures $11 \mathrm{a}$ and $\mathrm{b}$ present the polar diagrams of EDW wave height limit hwlimit for NWT-Dock60 and CWT-Dock60.

\section{FLOATING DOCKV GLOBAL STRENGHT ANALYSIS IN HEAD WAVES BY NUMERICAL EQUIVALENT DOCK BEAM MODEL}

The global strength analysis by equivalent beam model, under head wave, of the DOCKV version, using P_ACAVD [3] program, with modules 4.1-4.4 from Fig.1, lead to the global strength and free board criteria check in Tables 5.a-g.

Figs.12.a,b present the VBM, VSF diagrams during docking transition for shipyard case, for $L_{d}=0 \div 122.79 \mathrm{~m}$ (still water) over the pontoon deck, and Figs.12.c-f at head wave $(\mu=0)$ for DOCK D19747 $\mathrm{t}$ case, $L_{d \max }=122.79 \mathrm{~m}$.

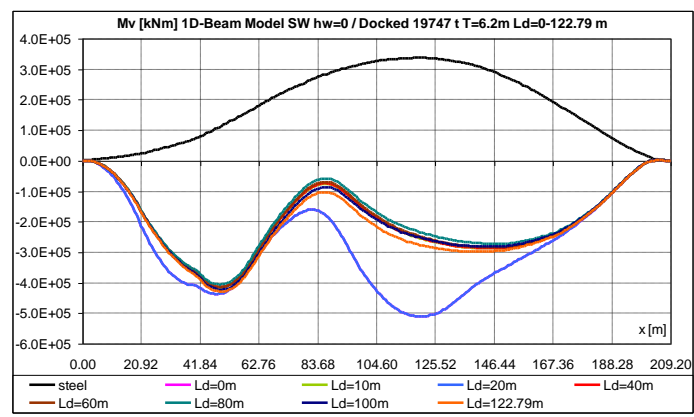

Fig. 12a. VBM, $L_{d}=0 \div 122.79 \mathrm{~m}, \mathrm{sw}, \mu=0$

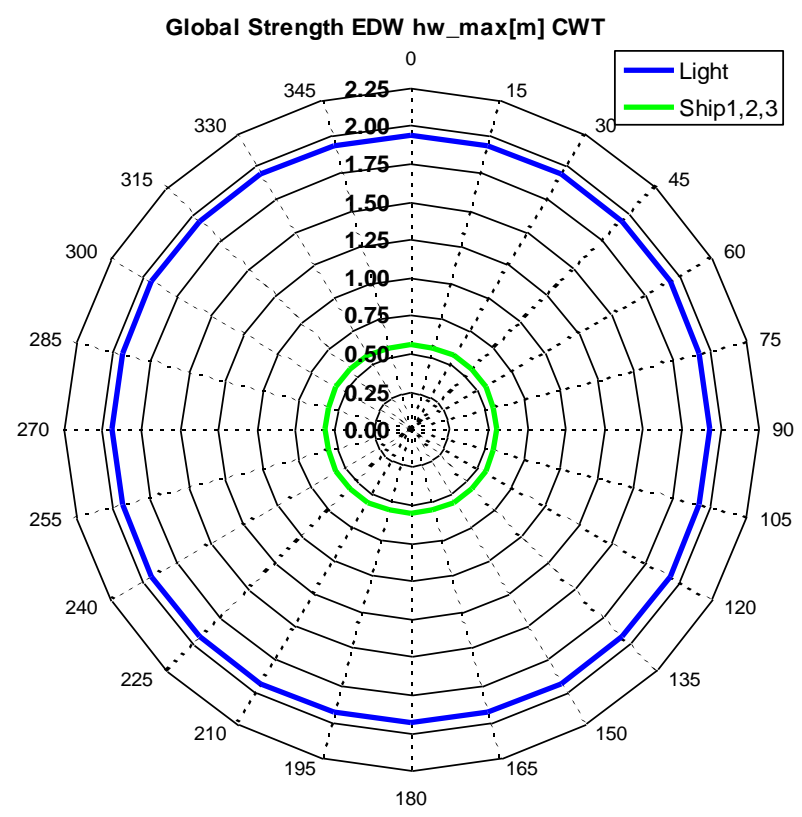

Fig. 11b. CWT-Dock60 polar diagram of EDW wave height limit $h_{\text {wlimit, }}$ all four loading cases, beam model

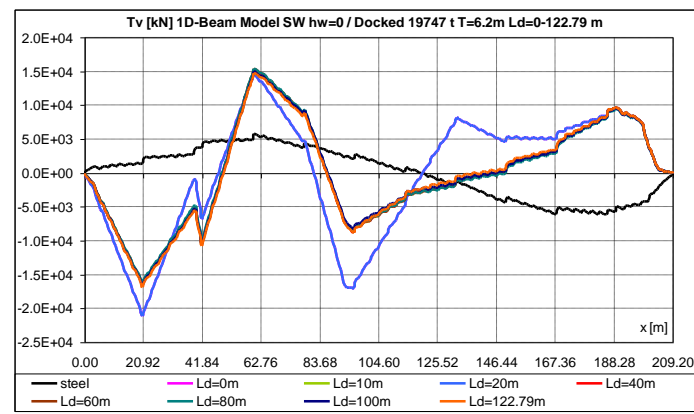

Fig. 12b. VSF, $L_{d}=0 \div 122.79 \mathrm{~m}$, sw, $\mu=0$

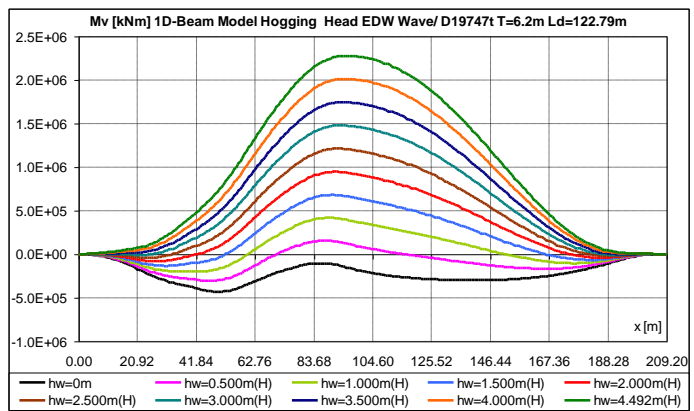

Fig. 12c VBM, D19747t, hogg., $\mu=0$

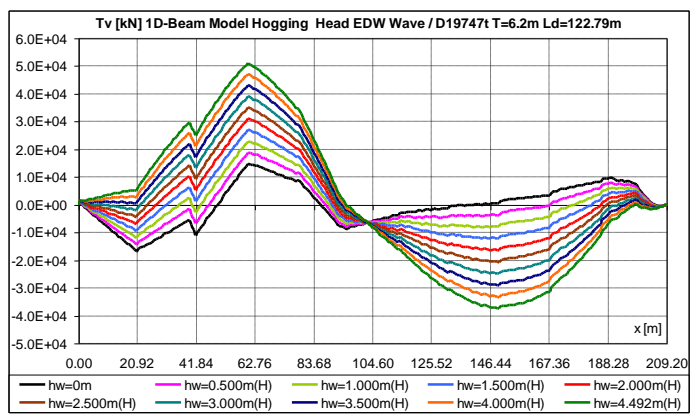

Fig. 12d. VSF, D19747t, hogg., $\mu=0$ 


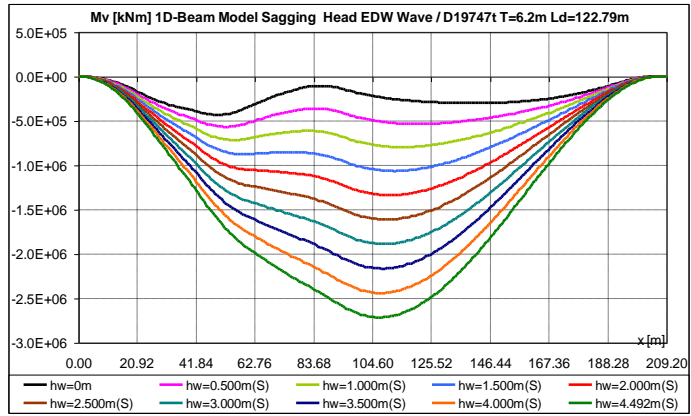

Fig. 12e. VBM, D19747t, sagg., $\mu=0$

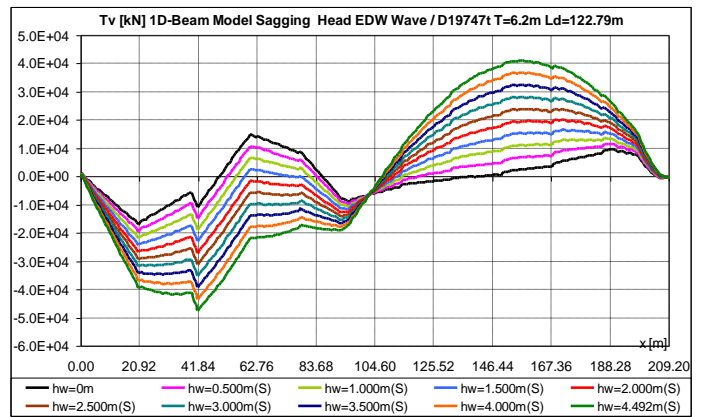

Fig. 12f. VSF, D19747t, sagg., $\mu=0$

Table 5a. Free board criterion, DOCKV $T_{m}=6.2 \mathrm{~m}, F_{s}=0.300 \mathrm{~m}$

\begin{tabular}{|c|c|c|c|c|c|c|c|c|c|c|c|}
\hline & & & & & & & & & & & \\
\hline EDW & $h_{w}[\mathrm{~m}]$ & 0 & 0.500 & 1.000 & 1.500 & 2.000 & 2.500 & 3.000 & 3.500 & 4.000 & 4.492 \\
\hline \multirow{10}{*}{ 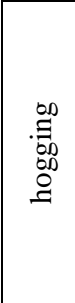 } & $T_{m}[\mathrm{~m}]$ & 6.200 & 6.191 & 6.182 & 6.174 & 6.165 & 6.156 & 6.147 & 6.138 & 6.128 & 6.118 \\
\hline & $\theta[\mathrm{rad}]$ & 0.00000 & -0.00028 & -0.00058 & -0.00089 & -0.00120 & -0.00151 & -0.00182 & -0.00213 & -0.00244 & -0.00275 \\
\hline & $x_{F}[\mathrm{~m}]$ & 100.104 & 100.107 & 100.110 & 100.114 & 100.117 & 100.120 & 100.124 & 100.128 & \begin{tabular}{|l|l|}
100.132 \\
\end{tabular} & 100.135 \\
\hline & $T_{p p}[\mathrm{~m}]$ & 6.200 & 6.219 & 6.240 & 6.263 & 6.285 & 6.307 & 6.330 & 6.351 & 6.372 & 6.394 \\
\hline & $T_{p v}[\mathrm{~m}]$ & 6.200 & 6.160 & 6.119 & 6.077 & 6.034 & 5.992 & 5.949 & 5.905 & 5.862 & 5.819 \\
\hline & $F_{a f t}[\mathrm{~m}]$ & 3.900 & 4.131 & 4.360 & 4.587 & 4.815 & 5.043 & 5.270 & 5.499 & 5.728 & 5.952 \\
\hline & $F_{m}[\mathrm{~m}]$ & 3.900 & 3.661 & 3.420 & 3.180 & 2.940 & 2.700 & 2.461 & 2.222 & 1.983 & 1.748 \\
\hline & $F_{\text {fore }}[\mathrm{m}]$ & 3.900 & 4.190 & 4.481 & 4.773 & 5.066 & 5.358 & 5.651 & 5.945 & 6.238 & 6.527 \\
\hline & $F_{\min }[\mathrm{m}]$ & 3.900 & 3.661 & 3.420 & 3.180 & 2.940 & 2.700 & 2.461 & 2.222 & 1.983 & 1.748 \\
\hline & $F_{\min } / F_{s}$ & $>1$ & $>1$ & $>1$ & $>1$ & $>1$ & $>1$ & $>1$ & $>1$ & $>1$ & $>1$ \\
\hline \multirow{10}{*}{ 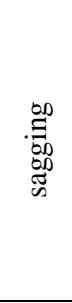 } & $T_{m}[\mathrm{~m}]$ & 6.200 & 6.207 & 6.215 & 6.222 & 6.229 & 6.236 & 6.243 & 6.250 & 6.256 & 6.262 \\
\hline & $\theta[\mathrm{rad}]$ & 0.00000 & 0.00032 & 0.00063 & 0.00095 & 0.00126 & 0.00157 & 0.00189 & 0.00220 & 0.00251 & 0.00282 \\
\hline & $x_{F}[\mathrm{~m}]$ & 100.104 & 100.100 & 100.097 & 100.094 & 100.092 & 100.089 & 100.086 & 100.084 & \begin{tabular}{|c|}
100.081 \\
\end{tabular} & 100.079 \\
\hline & $T_{p D}[\mathrm{~m}]$ & 6.200 & 6.175 & 6.152 & 6.127 & 6.103 & 6.079 & 6.054 & 6.029 & 6.005 & 5.980 \\
\hline & $T_{p v}[\mathrm{~m}]$ & 6.200 & 6.242 & 6.284 & 6.326 & 6.367 & 6.407 & 6.449 & 6.490 & 6.530 & 6.570 \\
\hline & $F_{a f t}[\mathrm{~m}]$ & 3.900 & 3.675 & 3.448 & 3.223 & 2.997 & 2.771 & 2.546 & 2.321 & 2.095 & 1.874 \\
\hline & $F_{m}[\mathrm{~m}]$ & 3.900 & 4.142 & 4.382 & 4.624 & 4.865 & 5.107 & 5.349 & 5.591 & 5.833 & 6.071 \\
\hline & $F_{\text {for }}[\mathrm{m}]$ & 3.900 & 3.608 & 3.316 & 3.024 & 2.733 & 2.443 & 2.151 & 1.860 & 1.570 & 1.284 \\
\hline & $F_{\min }[\mathrm{m}]$ & 3.900 & 3.608 & 3.316 & 3.024 & 2.733 & 2.443 & 2.151 & 1.860 & 1.570 & 1.284 \\
\hline & $F_{\min } / F_{s}$ & $>1$ & $>1$ & $>1$ & $>1$ & $>1$ & $>1$ & $>1$ & $>1$ & $>1$ & $>1$ \\
\hline
\end{tabular}

Table 5b. Global strength criteria, DOCKV light displacement, $T_{m}=6.2 \mathrm{~m}$ (with ballast)

\begin{tabular}{|c|c|c|c|c|c|c|c|c|c|c|c|}
\hline DW & & 0 & & & & & .500 & .0 & 3.500 & 4.000 & 4.492 \\
\hline \multirow{4}{*}{$\begin{array}{l}00 \\
00 \\
0\end{array}$} & $\mathrm{VBM}_{\max }$ & $5.11 \mathrm{E}+05$ & $3.26 \mathrm{E}+05$ & $3.41 \mathrm{E}+05$ & $5.96 \mathrm{E}+05$ & $8.53 \mathrm{E}+05$ & $1.11 \mathrm{E}+06$ & $1.37 \mathrm{E}+06$ & $1.63 \mathrm{E}+06$ & $1.89 \mathrm{E}+06$ & $2.15 \mathrm{E}+0$ \\
\hline & max/adm & 0.15 & 0.09 & 0.10 & 0.17 & 0.25 & 0.32 & 0.40 & 0.47 & 0.55 & 0.63 \\
\hline & $\mathrm{VSF}_{\text {max }}$ & $2.11 \mathrm{E}+04$ & $87 \mathrm{E}+04$ & $27 \mathrm{E}+04$ & $68 \mathrm{E}+04$ & $.08 \mathrm{E}+0$ & $.48 \mathrm{E}+04$ & $89 \mathrm{E}+0$ & $.29 \mathrm{E}+04$ & $4.69 \mathrm{E}+04$ & $.08 \mathrm{E}+$ \\
\hline & max/adm & 0.37 & 0.33 & 0.40 & 0.47 & 0.54 & 0.61 & 0.68 & 0.75 & 0.82 & 0.89 \\
\hline & & $5.11 \mathrm{E}+05$ & $7.70 \mathrm{E}+05$ & $1.03 \mathrm{E}+06$ & $1.30 \mathrm{E}+06$ & $1.57 \mathrm{E}+06$ & $1.84 \mathrm{E}+06$ & $2.12 \mathrm{E}+06$ & $2.39 \mathrm{E}+06$ & $2.67 \mathrm{E}+06$ & $2.94 \mathrm{E}+0$ \\
\hline & max/adm & 0.15 & 0.22 & 0.30 & 0.38 & 0.46 & 0.54 & 0.62 & 0.70 & 0.77 & 0.85 \\
\hline & $\mathrm{VSF}_{\mathrm{n}}$ & $2.11 \mathrm{E}+04$ & $2.36 \mathrm{E}+04$ & $2.60 \mathrm{E}+04$ & $2.85 \mathrm{E}+04$ & $3.11 \mathrm{E}+04$ & $3.36 \mathrm{E}+04$ & $3.61 \mathrm{E}+04$ & $3.86 \mathrm{E}+04$ & $4.11 \mathrm{E}+04$ & $4.40 \mathrm{E}+04$ \\
\hline & max/adm & 0.37 & 0.41 & 0.46 & 0.50 & 0.54 & 0.59 & 0.63 & 0.68 & 0.72 & 0.77 \\
\hline
\end{tabular}

Table 5c. Global strength criteria, DOCKV docking $19747 \mathrm{t}$ (and ballast)

$L d=0 \div 122.79 \mathrm{~m} h_{w}=0 \mathrm{~m} T_{m}=6.2 \mathrm{~m}$

\begin{tabular}{|c|c|c|c|c|c|c|c|c|c|c|}
\hline \multicolumn{2}{|c|}{$L d[\mathrm{~m}]$} & steel & 0 & 10 & 20 & 40 & 60 & 80 & 100 & 122.79 \\
\hline \multirow{4}{*}{$\begin{array}{l}\frac{\pi}{0} \\
\frac{0}{\partial}\end{array}$} & $\mathrm{VBM}_{\max }$ & $3.37 \mathrm{E}+05$ & $5.11 \mathrm{E}+05$ & $4.14 \mathrm{E}+05$ & $5.11 \mathrm{E}+05$ & $4.13 \mathrm{E}+05$ & $4.15 \mathrm{E}+05$ & $4.06 \mathrm{E}+05$ & $4.23 \mathrm{E}+05$ & $4.29 \mathrm{E}+05$ \\
\hline & $\max$ & & 0.149 & & 0.149 & & 0.121 & & 0.123 & 0.125 \\
\hline & $\mathrm{VSH}$ & $.13 \mathrm{E}+03$ & -04 & .64 & 04 & .6 & .65 & 04 & -04 & 104 \\
\hline & max/adm & 0.108 & 0.370 & 0.288 & 0.370 & 0.287 & 0.289 & 0.284 & 0.291 & 0.294 \\
\hline
\end{tabular}

Table 5d. Global strength criteria, DOCKV docking D19747 t (and ballast), $L d_{\max }=122.79 \mathrm{~m} T_{m}=6.2 \mathrm{~m}$

\begin{tabular}{|c|c|c|c|c|c|c|c|c|c|c|c|}
\hline$\overline{\mathrm{DW}}$ & $h_{w}[\mathrm{~m}]$ & 0 & 0.500 & 1.000 & 1.500 & 2.000 & 2.500 & 3.000 & 3.500 & 4.000 & 4.492 \\
\hline \multirow{4}{*}{ 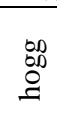 } & $\mathrm{VBM}_{\mathrm{m}}$ & $4.29 \mathrm{E}+05$ & $3.07 \mathrm{E}+05$ & $4.18 \mathrm{E}+05$ & $6.82 \mathrm{E}+05$ & $9.47 \mathrm{E}+05$ & $.21 \mathrm{E}+06$ & $1.48 \mathrm{E}+06$ & $.75 \mathrm{E}+06$ & $2.01 \mathrm{E}+06$ & $28 \mathrm{E}+06$ \\
\hline & $\mathrm{max} / \mathrm{adm}$ & 0.12 & 0.09 & 0.12 & 0.20 & 0.28 & 0.35 & 0.43 & 0.51 & 0.58 & 0.66 \\
\hline & $\mathrm{VSF}_{\text {max }}$ & $.68 \mathrm{E}+04$ & $.88 \mathrm{E}+04$ & $2.29 \mathrm{E}+04$ & $2.69 \mathrm{E}+04$ & $3.09 \mathrm{E}+04$ & $3.50 \mathrm{E}+04$ & $3.90 \mathrm{E}+04$ & $4.30 \mathrm{E}+04$ & $4.70 \mathrm{E}+04$ & $0 \mathrm{E}+04$ \\
\hline & $\mathrm{max} / \mathrm{adm}$ & 0.29 & 0.33 & 0.40 & 0.47 & 0.54 & 0.61 & 0.68 & 0.75 & 0.83 & 0.89 \\
\hline & VBN & $.29 \mathrm{E}+0$ & $5.65 \mathrm{E}+05$ & $7.95 \mathrm{E}+05$ & $1.06 \mathrm{E}+06$ & $34 \mathrm{E}+06$ & $61 \mathrm{E}+06$ & $89 \mathrm{E}+06$ & $2.16 \mathrm{E}+06$ & $2.44 \mathrm{E}+06$ & $71 \mathrm{E}+06$ \\
\hline & max/adm & 0.12 & 0.16 & 0.23 & 0.31 & 0.39 & 0.47 & 0.55 & 0.63 & 0.71 & 0.79 \\
\hline & $\mathrm{VSF}_{\max }$ & $1.68 \mathrm{E}+04$ & $1.92 \mathrm{E}+04$ & $2.17 \mathrm{E}+04$ & $2.42 \mathrm{E}+04$ & $2.69 \mathrm{E}+04$ & $3.10 \mathrm{E}+04$ & $3.51 \mathrm{E}+04$ & $3.92 \mathrm{E}+04$ & $4.32 \mathrm{E}+04$ & $4.73 E+04$ \\
\hline & $\mathrm{max} / \mathrm{adm}$ & 0.29 & 0.34 & 0.38 & 0.42 & 0.47 & 0.54 & 0.62 & 0.69 & 0.76 & 0.83 \\
\hline
\end{tabular}


Table 5e. Global strength criteria, DOCKV D27000 t (\& ballast), docked ship 1 uniform-mass distribution, $T_{m}=6.2 \mathrm{~m}$

\begin{tabular}{|c|c|c|c|c|c|c|c|c|c|c|c|}
\hline $\mathrm{DW}$ & $h_{w}[\mathrm{~m}]$ & 0 & 0.500 & 1.000 & 1.500 & 2.000 & 2.500 & 3.000 & 3.213 & 3.908 & $\frac{m-0 .}{4.492}$ \\
\hline \multirow{4}{*}{$\begin{array}{l}500 \\
00 \\
0 \\
\varrho\end{array}$} & $\mathrm{VBM}_{\mathrm{m}}$ & $.28 \mathrm{E}+06$ & $1.01 \mathrm{E}+06$ & $7.40 \mathrm{E}+05$ & $5.72 \mathrm{E}+05$ & $4.34 \mathrm{E}+05$ & $3.07 \mathrm{E}+05$ & $4.12 \mathrm{E}+05$ & $5.24 \mathrm{E}+05$ & $3.92 \mathrm{E}+05$ & $20 \mathrm{E}+06$ \\
\hline & max/adm & 0.37 & 0.29 & 0.22 & 0.17 & 0.13 & 0.09 & 0.12 & 0.15 & 0.26 & 0.35 \\
\hline & VSF & $3.09 \mathrm{E}+04$ & $2.69 \mathrm{E}+04$ & $2.29 \mathrm{E}+04$ & $1.89 \mathrm{E}+04$ & $.49 E+04$ & $1.89 \mathrm{E}+04$ & $2.29 \mathrm{E}+04$ & $2.46 \mathrm{E}+04$ & $3.02 \mathrm{E}+04$ & $3.49 \mathrm{E}+04$ \\
\hline & $\max / c$ & 0.54 & 0.47 & 0.40 & 0.33 & 0.26 & 33 & 0.40 & 0.43 & 0.53 & 0.61 \\
\hline & VBN & $28 \mathrm{E}+06$ & $.55 \mathrm{E}+06$ & $.83 \mathrm{E}+06$ & $2.10 \mathrm{E}+06$ & $2.38 \mathrm{E}+06$ & $2.66 \mathrm{E}+06$ & $.93 \mathrm{E}+06$ & $3.05 \mathrm{E}+06$ & $3.44 \mathrm{E}+06$ & $77 \mathrm{E}+\mathrm{c}$ \\
\hline & max/adm & 0.37 & 0.45 & 0.53 & 0.61 & 0.69 & 0.77 & 0.85 & 0.89 & 1.00 & 1.09 \\
\hline & $\mathrm{VSF}_{\max }$ & $3.09 \mathrm{E}+04$ & $3.49 \mathrm{E}+04$ & $3.90 \mathrm{E}+04$ & $4.30 \mathrm{E}+04$ & $4.71 \mathrm{E}+04$ & $5.12 \mathrm{E}+04$ & $5.53 E+04$ & $5.70 \mathrm{E}+04$ & $6.27 \mathrm{E}+04$ & $6.75 \mathrm{E}+04$ \\
\hline & max/adm & 0.54 & 0.61 & 0.68 & 0.76 & 0.83 & 0.90 & 0.97 & 1.00 & 1.10 & 1.18 \\
\hline
\end{tabular}

Table 5f. Global strength criteria, DOCKV D27000 t (and ballast), docked ship 2 hogging-mass distribution, $T_{m}=6.2 \mathrm{~m}$

\begin{tabular}{|c|c|c|c|c|c|c|c|c|c|c|c|}
\hline & $\mathrm{Iml}$ & & 056 & & & & 2500 & 3000 & 3760 & $\frac{1000}{4000}$ & 4.492 \\
\hline \multirow{4}{*}{$\begin{array}{l}0 \\
0 \\
0\end{array}$} & $\frac{n_{w 1}}{\mathrm{VBN}}$ & $9.91 \mathrm{E}+05$ & $7.67 \mathrm{E}+05$ & $5.61 \mathrm{E}+05$ & $.84 \mathrm{E}+05$ & $4.97 \mathrm{E}+05$ & $7.55 \mathrm{E}+05$ & $.01 \mathrm{E}+06$ & $.42 \mathrm{E}+06$ & $.54 \mathrm{E}+06$ & $\begin{array}{l}.7 .49 \mathrm{E}+06 \\
.79 \mathrm{E}+ \\
\end{array}$ \\
\hline & $\max / \operatorname{madm}$ & 0.29 & 0.22 & 0.16 & 0.11 & 0.14 & 0.22 & 0.29 & 0.41 & 0.45 & 0.52 \\
\hline & $\mathrm{VSF}_{\text {max }}$ & $71 \mathrm{E}+$ & $32 \mathrm{E}+0$ & $93 \mathrm{E}+1$ & $30 \mathrm{E}$ & $70 \mathrm{E}$ & $11 \mathrm{E}+$ & & $13 \mathrm{H}$ & $32 \mathrm{E}$ & $71 \mathrm{E}+1$ \\
\hline & max/adm & 0.48 & 0.41 & 0.34 & 0.40 & 0.47 & 0.55 & 0.62 & 0.72 & 0.76 & 0.83 \\
\hline & VBM & $9.91 \mathrm{E}$ & $.23 \mathrm{E}$ & $.47 \mathrm{E}-$ & $73 \mathrm{E}$ & $99 \mathrm{E}+06$ & $25 \mathrm{E}+06$ & $.52 \mathrm{E}$ & $2.93 \mathrm{E}+06$ & 06 & $33 \mathrm{E}+06$ \\
\hline & max/adm & 0.29 & 0.3 & 0.43 & 0.50 & & 0.66 & 0 . & 0.8 & 0.89 & 0.97 \\
\hline & $\mathrm{VSF}_{\mathrm{m}}$ & $2.71 \mathrm{E}+04$ & $3.10 \mathrm{E}+04$ & $3.50 \mathrm{E}+04$ & $3.89 \mathrm{E}+04$ & $4.29 \mathrm{E}+04$ & $4.69 \mathrm{E}+04$ & $5.09 \mathrm{E}+04$ & $5.70 \mathrm{E}+04$ & $5.89 \mathrm{E}+04$ & $28 \mathrm{E}+04$ \\
\hline & max/adm & 0.48 & 0.54 & 0.61 & 0.68 & 0.75 & 0.82 & 0.8 & 1.00 & 1.03 & 1.10 \\
\hline
\end{tabular}

Table 5g. Global strength criteria, DOCKV D27000 t (and ballast), docked ship 3 sagging-mass distribution, $T_{m}=6.2 \mathrm{~m}$

\begin{tabular}{|c|c|c|c|c|c|c|c|c|c|c|c|}
\hline & & 0 & & & 50 & & 2107 & & & & 4492 \\
\hline \multirow{4}{*}{$\begin{array}{l}600 \\
00 \\
0 \\
0\end{array}$} & $\frac{n_{w[111]}}{\mathrm{VBM}_{\max }}$ & $.68 \mathrm{E}+06$ & $.40 \mathrm{E}+06$ & $.13 \mathrm{E}+06$ & $9.35 \mathrm{E}+05$ & $\frac{2.000}{7.80 \mathrm{E}+05}$ & 7.21E+05 & $5.00 \mathrm{E}+05$ & $4.56 \mathrm{E}+05$ & $5.11 \mathrm{E}+05$ & $\begin{array}{c}.7 .492 \\
.77 \mathrm{E}+05\end{array}$ \\
\hline & \begin{tabular}{|l}
$\mathrm{max} / \mathrm{adm}$ \\
\end{tabular} & 0.49 & 0.41 & 0.33 & 0.27 & 0.23 & 0.21 & 0.15 & 0.13 & 0.15 & 0.23 \\
\hline & $\mathrm{VSF}_{\text {max }}$ & $2 \mathrm{E}$ & $52 \mathrm{E}+04$ & $1 \mathrm{E}+\mathrm{c}$ & $71 \mathrm{E}+04$ & $31 \mathrm{E}+04$ & $15 \mathrm{E}+04$ & $73 E+04$ & $87 \mathrm{E}$ & $54 E+04$ & $\overline{93 E+04}$ \\
\hline & max/adm & 0.69 & 0.62 & 0.55 & 0.48 & 0.41 & 0.38 & 0.30 & 0.33 & 0.44 & 0.51 \\
\hline & & $1.68 \mathrm{E}$ & $1.96 \mathrm{E}+06$ & $2.23 \mathrm{E}+06$ & $2.51 \mathrm{E}+06$ & $2.79 \mathrm{E}+06$ & $2.90 \mathrm{E}+06$ & $3.34 \mathrm{E}+06$ & $3.44 \mathrm{E}+06$ & $3.90 \mathrm{E}$ & $1.17 \mathrm{E}+0$ \\
\hline & \begin{tabular}{|l}
$\max / \mathrm{adm}$ \\
\end{tabular} & 0.49 & 0.57 & 0.65 & 0.73 & 0.81 & 0.84 & 0.97 & 1.00 & 1.13 & 1.21 \\
\hline & $\mathrm{VSF}_{\mathrm{ma}}$ & $.92 \mathrm{E}+04$ & $4.32 \mathrm{E}+04$ & $4.73 E+04$ & $5.13 E+04$ & $5.54 \mathrm{E}+04$ & $5.70 \mathrm{E}+04$ & $6.35 \mathrm{E}+04$ & $6.50 \mathrm{E}+04$ & $7.17 \mathrm{E}+04$ & $.57 \mathrm{E}+04$ \\
\hline & \begin{tabular}{|l|}
$\mathrm{max} / \mathrm{adm}$ \\
\end{tabular} & 0.69 & 0.76 & 0.83 & 0.90 & 0.97 & 1.00 & 1.11 & 1.14 & 1.26 & 1.33 \\
\hline
\end{tabular}

Table 6. The small floating docks NWT-Dock60 and CWT-Dock60 results, in oblique waves, by equivalent beam model

\begin{tabular}{|c|c|c|c|c|c|c|c|c|}
\hline Docking case & NWT Light & NWT Ship 1 & NWT Ship 2 & NWT Ship 3 & CWT Light & CWT Ship 1 & CWT Ship 2 & CWT Ship 3 \\
\hline$h_{\mathrm{w} \text { limit }}[\mathrm{m}]$ & 0.640 & 0.420 & 0.420 & 0.261 & 1.930 & 0.550 & 0.550 & 0.550 \\
\hline criterion & strength & free board & free board & strength & free board limitations at pontoon main deck \\
\hline inland & IN $(0.64)$ & IN $(0.42)$ & IN $(0.42)$ & SW & IN $(1.93)$ & IN $(0.55)$ & IN $(0.55)$ & IN $(0.55)$ \\
\hline costal & \multicolumn{3}{|c|}{ sheltered operation } & & RE $(37 \%)$ & \multicolumn{3}{c|}{ sheltered operation } \\
\hline
\end{tabular}

Table 7. DOCKV large floating dock results by equivalent beam models and head design waves

\begin{tabular}{|c|c|c|c|c|c|}
\hline Docking case & Light $T 6.2$ & D19747t T6.2 & D27000t hogg. T6.2 & D27000t unif. T6.2 & D27000t sagg. T6.2 \\
\hline$h_{\mathrm{w} \text { limit }}[\mathrm{m}]$ & 4.492 & 4.492 & 3.769 & 3.213 & 2.197 \\
\hline criterion & \multicolumn{2}{|c|}{ no restrictions } & AVSF admissible global strength, sagging EDW condition \\
\hline inland & $\mathrm{IN}(2.0)$ & $\mathrm{IN}(2.0)$ & $\mathrm{IN}(2.0)$ & $\mathrm{IN}(2.0)$ & $\mathrm{IN}(2.0)$ \\
\hline costal & $\mathrm{RE}(50 \%)$ & $\mathrm{RE}(50 \%)$ & $\approx \mathrm{RE}(40 \%)$ & $\approx \operatorname{RE}(35 \%)$ & $\approx \operatorname{RE}(24 \%)$ \\
\hline
\end{tabular}

\section{CONCLUSIONS}

The study of three floating docks versions leads to the following results:

1) Both small size floating docks Dock60 have a structural enhancement by considering the frames distance $a_{F r}=a_{0}=600 \mathrm{~mm}$, having am increased global ultimate strength (Fig.3.b, Fig.5.b, Table 2) as in initial versions [3].

2) For small size floating dock NWT-dock60, with non-continuous side ballast tanks, function to the heading angle $\mu=0-90^{\circ}$ (360), the following limit wave heights are recorded: light case (Table 3a) $h_{\text {wlim }}=0.640-1.278 \mathrm{~m}$ (strength criteria VBM hogging, $\mu=0 \div 45^{\circ}$ ) and $h_{\text {wlim }}=1.800 \mathrm{~m}$ (free board criteria, $\mu=60 \div 90^{\circ}$ ); ship 1 and ship 2 cases (Tables $3 \mathrm{~b}, \mathrm{c}$ ) $h_{\text {wlim }}=0.420 \mathrm{~m}$ (free board criteria, $\mu=0 \div 90^{\circ}$ ); ship 3 case (Table 3d) $h_{\text {wlim }}=0.261-0.318 \mathrm{~m}$ (strength criteria VBM hogging, $\mu=0 \div 30^{\circ}$ ) and $h_{\text {wlim }}=0.420 \mathrm{~m}$ (free board criteria, $\mu=45 \div 90^{\circ}$ ). The synthesis of the operation limits for the NWT-dock60 is presented in the polar diagram of oblique EDW height (Fig. 11a) and Table 6.

3) For small size floating dock CWT-dock60, with continuous side ballast tanks, function to the heading angle $\mu=0-90^{\circ}$ (360) the following limit wave heights are recorded: light case (Table 4a) $h_{\text {wlim }}=1.930 \mathrm{~m}$, ship 1, 2, 3 cases (Tables 4b, c, d) $h_{\text {wlim }}=0.550 \mathrm{~m}$, with restrictions only due to the free board criterion $\left(\mu=0 \div 90^{\circ}\right)$. The synthesis of the operation limits for the CWT-dock60 is presented in the polar diagram of oblique EDW height (Fig. 11b) and Table 6. 
4) For both floating docks Dock60 the most restrictive wave case is the head (follow) $\mu=0^{\circ}\left(180^{\circ}\right)$ condition, having a uniform structure over the whole length and no stress hot-spots included in the equivalent beam dock models. The NWT-Dock60 version has operation condition restrictions for inland $\mathrm{SW} \div \mathrm{IN}(0.64)$ and costal must be sheltered. The CWT-Dock60 version has less operation restrictions for inland $\mathrm{IN}(0.55) \div \mathrm{IN}(1.93)$ and costal can be relocated in light case RE(37\%) with special approval, but must operate in sheltered conditions in any docking cases (Figs. 11a, b, Table 6). Although NWT version has more operation restrictions, it is recommended for inland harbours, being possible easier to be constructed by a conversion of a standard barge or pontoon.

5) For the large floating DOCKV [2], no restrictions are obtained by free board criterion (Table 5a). In light (Table 5b) case and during the shipyard docking scenario D19747t (Tables 5c, d), no strength restrictions are recorded, so that $h_{\text {wlim }}=4.492$ $\mathrm{m}$ with operation class inland $\mathrm{IN}(2.0)$ and costal $\mathrm{RE}(50 \%)$. For extreme docking cases D27000 t, the strength limits are on VSF sagging (Tables 5e, f, g), resulting $h w_{\text {him }}=2.197 \div 3.769 \mathrm{~m}$, so that for inland operation are no restrictions $\mathrm{IN}(2.0)$ and for costal $\operatorname{RE}(24 \% \div 40 \%)$ requiring special approval. The synthesis of the operation limits for the DOCKV, head wave conditions are presented in Table 7. No oblique waves are considered, according to small size dock results.

6) Further studies shall include structural analysis on 3D-FEM models for the floating dock, so that the stress hot-spots can be considered, and the limits by strength criteria 3D stress state can be used.

\section{ACKNOWLEDGEMENT}

This study has been developed in the frame of the $\mathrm{PhD}$ topic "Studies concerning the analysis of a floating dock structure on extreme loads" and we express our thanks to the IOSUD-UDJG, PhD Engineering School from "Dunarea de Jos" University of Galati. The authors wish to express their thanks to VARD Tulcea Shipyard, who granted us the large floating dock technical data [2].

\section{REFERENCES}

[1] Bidoae R., Ionas O., 2004, Ship theory, Didactic and Pedagogic Publishing House, Bucharest (in Romanian).

[2] Burlacu E., 2017, Floating docks design state of art. Technical data for large floating dock from VARD Tulcea Shipyard, (PhD Report), "Dunarea de Jos" University, Galati (in Romanian).

[3] Burlacu E., Pacuraru F., Domnisoru L., 2017, On the development of design software for floating dock units operating capabilities analysis, Mechanical Testing and Diagnosis 7(1) 5-17.

[4] BV, 2018, Rules for the design and classification of ships, Floating docks, Bureau Veritas, Paris.

[5] DNVGL, 2018, Rules for floating docks. Poseidon program, Det Norske Veritas, Hovik, Germanischer Lloyd, Hamburg.

[6] Domnisoru L., Modiga A, Gasparotti C., 2016, Global strength assessment in oblique waves of a large gas carrier ship, ModTech, IOP Materials Science and Engineering (145) Article 082009.

[7] Eyres D.J, 2007, Ship construction, ButterworthHeinemann, Oxford.

[8] Hughes O.F., 2000, Ship structural design. A rationallybased, computer-aided optimization approach, SNAME, Wiley \& Sons, N.Y.

[9] ISSC, 2018, International Ships and Offshore Structures Congress. Environment. Loads. Quasistatic response. Ultimate strength. Dynamic response. Design principles and criteria, IOS Press, Amsterdam.

[10] Papanikolaou A., 2014, Ship design. Methodology of preliminary design, Springer Verlag, Berlin. 